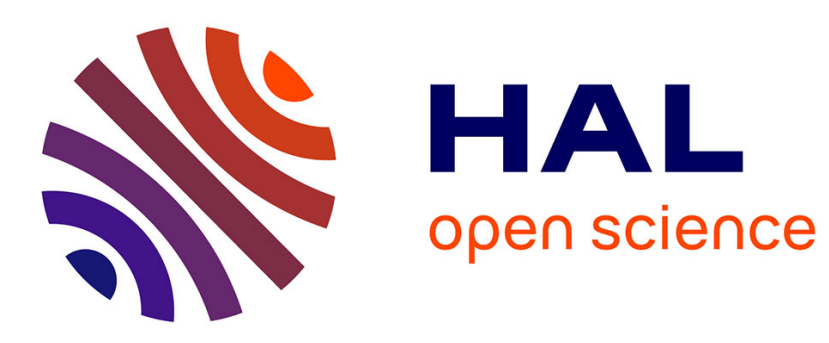

\title{
Background-Foreground Modeling Based on Spatiotemporal Sparse Subspace Clustering
}

Sajid Javed, Arif Mahmood, Thierry Bouwmans, Ki Jung Soon

\section{To cite this version:}

Sajid Javed, Arif Mahmood, Thierry Bouwmans, Ki Jung Soon. Background-Foreground Modeling Based on Spatiotemporal Sparse Subspace Clustering. IEEE Transactions on Image Processing, 2017, 26 (12), pp.5840 - 5854. 10.1109/TIP.2017.2746268 . hal-01580085

\section{HAL Id: hal-01580085 https://hal.science/hal-01580085}

Submitted on 11 Jan 2018

HAL is a multi-disciplinary open access archive for the deposit and dissemination of scientific research documents, whether they are published or not. The documents may come from teaching and research institutions in France or abroad, or from public or private research centers.
L'archive ouverte pluridisciplinaire HAL, est destinée au dépôt et à la diffusion de documents scientifiques de niveau recherche, publiés ou non, émanant des établissements d'enseignement et de recherche français ou étrangers, des laboratoires publics ou privés. 


\section{Background-Foreground Modeling Based on Spatiotemporal Sparse Subspace Clustering}

Article in IEEE Transactions on Image Processing · August 2017

DOI: $10.1109 /$ TIP.2017.2746268

\section{CITATIONS}

2

4 authors:

\section{Sajid Javed}

The University of Warwick

28 PUBLICATIONS 144 CITATIONS

SEE PROFILE

\section{Thierry Bouwmans}

Université de La Rochelle

82 PUBLICATIONS 1,690 CITATIONS

SEE PROFILE

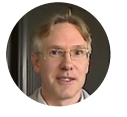

READS

198

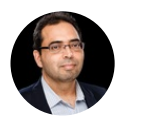

Arif Mahmood

University of Western Australia

50 PUBLICATIONS 393 CITATIONS

SEE PROFILE

Soon Ki Jung

Kyungpook National University

147 PUBLICATIONS 620 CITATIONS

SEE PROFILE

Some of the authors of this publication are also working on these related projects: 


\title{
Background-Foreground Modeling Based on Spatiotemporal Sparse Subspace Clustering
}

\author{
Sajid Javed, Arif Mahmood, Thierry Bouwmans, and Soon Ki Jung, Senior Member, IEEE,
}

\begin{abstract}
Background estimation and foreground segmentation are important steps in many high-level vision tasks. Many existing methods estimate background as a low-rank component and foreground as a sparse matrix without incorporating the structural information. Therefore, these algorithms exhibit degraded performance in the presence of dynamic backgrounds, photometric variations, jitter, shadows, and large occlusions. We observe that these backgrounds often span multiple manifolds. Therefore, constraints that ensure continuity on those manifolds will result in better background estimation. Hence, we propose to incorporate the spatial and temporal sparse subspace clustering into the RPCA framework. To that end, we compute a spatial and temporal graph for a given sequence using motion-aware correlation coefficient. The information captured by both graphs is utilized by estimating the proximity matrices using both the normalized Euclidean and geodesic distances. The low-rank component must be able to efficiently partition the spatiotemporal graphs using these Laplacian matrices. Embedded with the RPCA objective function, these Laplacian matrices constrain the background model to be spatially and temporally consistent, both on linear and nonlinear manifolds. The solution of the proposed objective function is computed by using the LADMAP optimization scheme. Experiments are performed on challenging sequences from five publicly available datasets and are compared with 23 existing state-of-the-art methods. The results demonstrate excellent performance of the proposed algorithm for both background estimation and foreground segmentation.
\end{abstract}

Index Terms-Background modeling, Foreground Detection, Graph Regularization, Subspace Clustering, Robust Principal Component Analysis.

\section{INTRODUCTION}

B ACKGROUND estimation and foreground segmentation originate in numerous applications in computer vision including moving object detection [65], video surveillance [4], visual object tracking [59], and salient motion detection [15]. Background modeling is mainly intended to efficiently and accurately extract a model which describes the scene without foreground objects. On the other hand, foreground detection is intended for segmenting moving objects from the known background model [2], [32], [35]. Both of these methods become challenging in the presence of dynamic background, changing lighting conditions, and jitter induced by the sensor. Background modeling also suffers in the presence of occlusion because of foreground objects. A number of techniques have been proposed in literature that mostly address relatively

S. Javed and S. K. Jung are with Virtual Reality Laboratory, the School of Computer Science and Engineering, Kyungpook National University, 80 Daehak-ro, Buk-gu, Daegu, 41566, Republic of Korea. (e-mail: sajid@ vr.knu.ac.kr, skjung@knu.ac.kr). (corresponding author: Soon Ki Jung)

A. Mahmood is with Department of Computer Science and Engineering, Qatar University, Qatar. (email: arif.mahmood@qu.edu.qa)

T. Bouwmans is with Universite de La Rochelle, Laboratoire Mathematiques, Image et Applications (MIA), 23 Avenue Albert Einstein, La Rochelle, BP 33060-17301, France. (email: tbouwman@univ-lr.fr)

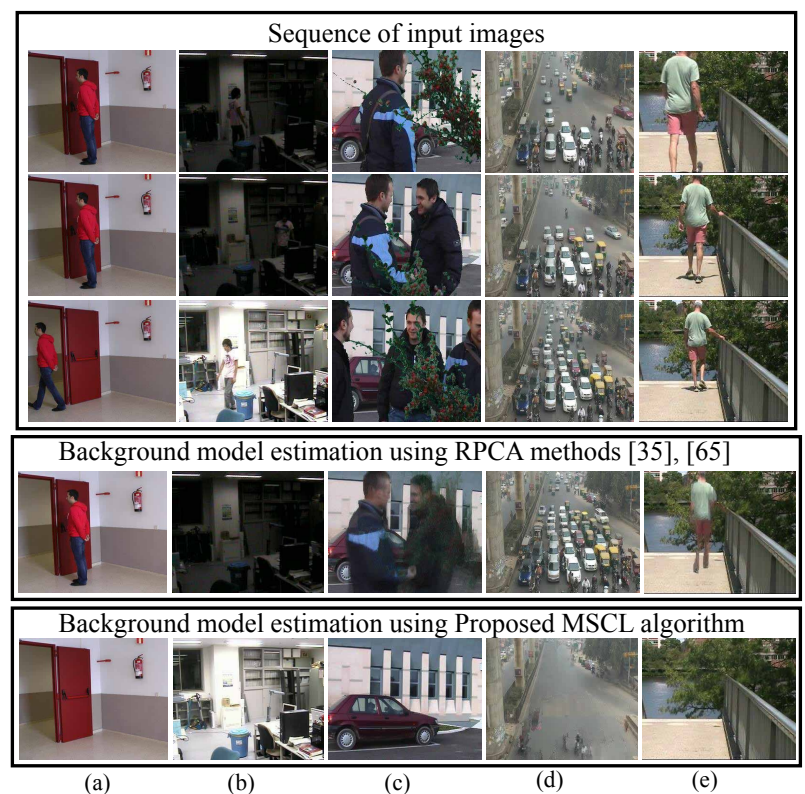

Fig. 1. Estimated background examples in the SBM.net dataset [37]: (a) Sequence 'IMB01 from the category 'intermittent object motion contains redundant frames. (b) Sequence "camera parameter from the category 'illumination changes. (c) Sequence 'People \& Foliage from the category "clutter has large occlusions. (d) Sequence "boulvardJam from the category "clutter has dominant foreground. (e) Sequence 'overpass from the category 'dynamic motion has always moving objects. In all of these cases, the proposed MSCL algorithm has produced significant improvement.

simple scenarios for the estimation of background and/or foreground component [2].

As an example, most methods perform well when the pixels or regions of the background are visible for longer periods in the training data. Furthermore, the performance of many methods [11], [31], [57] degrades significantly in real-time scenarios, especially if the background scene is visible for short periods. Moreover, the performance of foreground detection methods is also effected by the dynamic pixels, such as those caused by the rippling of water surface, swaying of trees, and sudden variations in lighting conditions. Recently, Robust Principal Component Analysis (RPCA) has been shown to be an efficient framework for segregating background and foreground components [7], [53]. In RPCA, Wright et al. [53] considered background-foreground modeling as a matrix decomposition problem:

$$
\min _{\mathbf{B}, \mathbf{F}}|| \mathbf{B}\left\|_{*}+\lambda_{1}\right\| \mathbf{F} \|_{1} \text { such that } \mathbf{X}=\mathbf{B}+\mathbf{F},
$$

where $\mathbf{X}=\left[\mathbf{x}_{1}, \mathbf{x}_{2}, \ldots, \mathbf{x}_{n}\right] \in \mathbb{R}^{p \times n}$ is the input video sequence of $n$ frames, and each $\mathbf{x}_{i} \in \mathbb{R}^{p}$ denotes $i$-th frame. The low-rank component $\mathbf{B}$ corresponds to the background model whereas the locally deforming regions called foreground constitute the sparse matrix $\mathbf{F}$. This decomposition is achieved by 


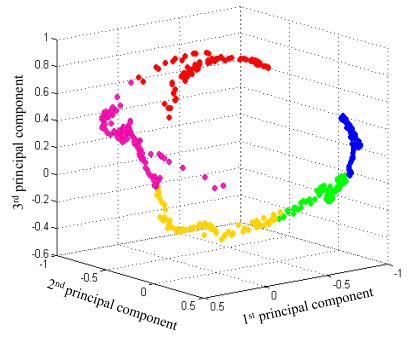

(a)

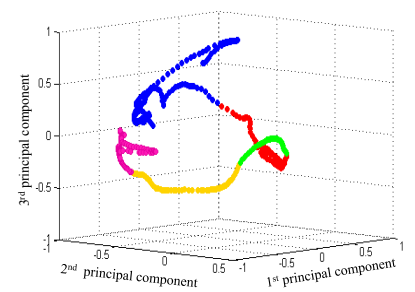

(c)

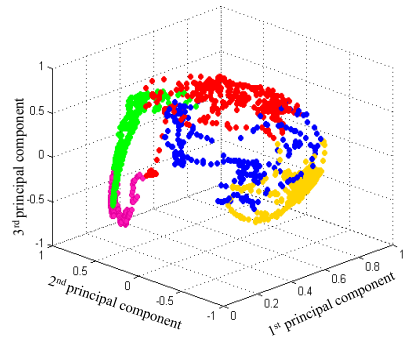

(b)

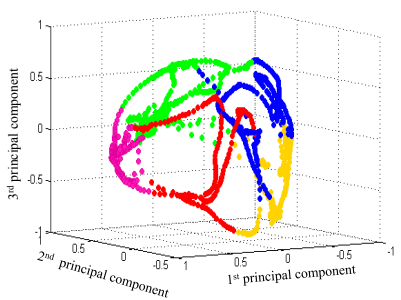

(d)
Fig. 2. (a) and (c) Temporal graphs of the dynamic background sequences 'Water Surface' and 'Campus' from the I2R dataset [26]. (b) and (d) Corresponding spatial graphs of the same sequences. The edges are removed to avoid clutter. These graphs are plotted using three major principal components of the geodesic space. The different colors indicate different clusters computed using subspace spectral clustering in the geodesic space. The shapes of different clusters as well as the individual graphs are nonlinear, indicating that the background sequences span multiple nonlinear manifolds.

solving a convex optimization method [7]. In (1), $\|\cdot\|_{*}$ denotes the nuclear norm, which is defined as the sum of all singular values, and $\|\cdot\|_{1}$ is the $l_{1}$-norm. Nuclear norm enforces the background images to be linearly correlated, and the $l_{1}$-norm constrains the foreground matrix to be sparse.

In many real scenarios, traditional RPCA methods [11], [35], [65] suffer from some obvious limitations. For example, many real-world scenarios contain redundant information in the form of motionless frames, where foreground objects remain static for long durations and then start moving. If the redundant information is not properly handled, the outlier regions will also appear in the estimated background (Fig. 1 (a)). Furthermore, sudden photometric variations of the background increase its rank; therefore, the low-rank background model estimation fails (Fig. 1 (b)). If both background and foreground coexist in each frame, the low-rank component cannot efficiently capture the background model because of abrupt changes (Fig. 1 (c)-(d)). In the case of some dynamic background sequences, the low-rank component fails to estimate the background model (Fig. 1 (e)). Given these common real-world scenarios, the existing methods fail to achieve good quality background-foreground models because of unrealistic assumptions.

We observe that the real-world background sequences, as shown in Fig. 1, may span one or more linear or nonlinear manifolds. To investigate this fact, we applied sparse subspace clustering on multiple dynamic background scenes. Nonlinear shapes of the clusters in 3D principal-space of geodesic distance may be observed in Fig. 2. In high dimensional space, the frames in each cluster span different nonlinear manifolds. Therefore, constraining the estimated low-rank B to remain consistent with the subspace structure of the original background significantly improves the background modeling performance.
Compared to the traditional RPCA techniques, following modifications are proposed in this study:

- Two graphs are constructed, namely, a temporal graph over the background frames and a spatial graph over the spatial background locations using motion-aware Correlation Coefficient (CC) as a similarity measure to handle the background modeling efficiently.

- For background models spanning linear subspaces, the normalized Euclidean distance based proximity matrices are directly computed from these graphs. Sparse coding is used to linearly decompose each column of each proximity matrix using the remaining columns as dictionary items. The linear coefficients are used to recalculate a new proximity matrix which is then used to compute the sparse subspace based normalized Laplacian matrix [12].

- For backgrounds spanning nonlinear manifolds, Geodesic Distance (GD) based proximity matrices are computed for both graphs. For each matrix, we apply sparse coding and compute the geodesic subspace based Laplacian matrix [34]. The normalized Laplacian matrices computed over both Euclidean and Geodesic distances are embedded into the RPCA framework.

These graph-based regularization's help us to overcome the limitations of the existing methods [7], [31], [35], [65]. To the best of our knowledge, this is the first study that integrates the spatiotemporal clustering information into low-rank component for improved background modeling.

We name the resulting algorithm as Motion-assisted Spatiotemporal Clustering of Low-rank (MSCL). In the proposed algorithm, we first detect a set of dynamic frames $\mathbf{D}$ within the sequence $\mathbf{X}$ by eradicating motionless frames, which help us in dealing with redundant data problem, as shown in Fig. 1 (a). For this purpose, we estimate the optical flow between the consecutive frames and use it to generate a binary motion mask, which assists us to obtain approximate knowledge on the pixels of background and foreground components. The spatial and temporal graphs are computed using motion-aware CC [33], which includes pixels exhibiting motion less than a predefined threshold. The use of CC-based regularization enforces the background model to be invariant to lighting condition variations and to enhance continuity on linear manifolds, while GD-based regularization ensures the continuity of the background model on nonlinear manifolds.

We solve our proposed objective function using the Linearized Alternating Direction Method with Adaptive Penalty (LADMAP) optimization because of its efficiency in using less auxiliary variables with convergence guarantee [28], [30]. We evaluated our proposed algorithm on a new dataset Scene Background Modeling.net (SBM.net) [37], which contains 8 challenging categories and 80 videos. We compared the proposed MSCL algorithm with several existing state-of-theart methods. Our proposed algorithm outperforms these earlier methods on almost all categories of the SBM.net dataset ${ }^{1}$.

Compared to existing methods, our algorithm generates better foreground model; however, we do not explicitly constrain the foreground, which may result in a noisy and dis-contiguous

\footnotetext{
${ }^{1}$ http://pione.dinf.usherbrooke.ca/results/
} 


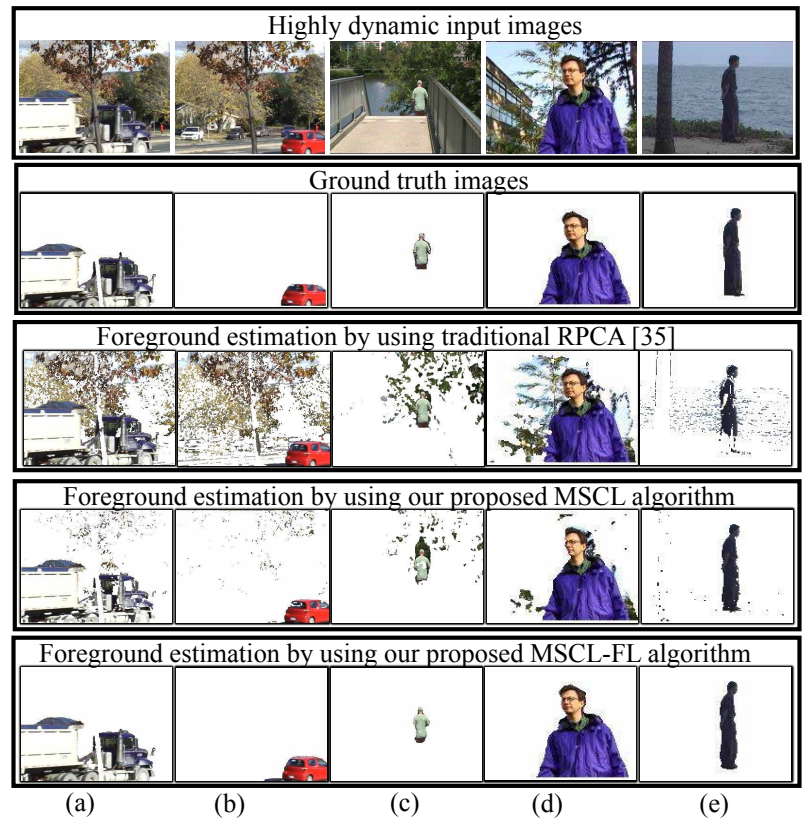

Fig. 3. Results of foreground detection. (a)-(b) 'Fall' sequence from the CDnet dataset, (c) 'Overpass' from the CDnet dataset, (d) 'Waving Trees' from the Wallflower dataset, and (e) 'Water Surface' the from I2R dataset. From top to bottom: (1) input images, (2) ground-truth images of the foreground, (3) foreground estimation using RPCA methods [35], (4) estimated foreground mask by using the proposed MSCL algorithm, and (5) results of the foreground estimation by using our proposed MSCL-FL algorithm.

foreground (Fig. 3). Figs. 3 (a)-(e) show that although our proposed algorithm estimated an outlier-free background model in the case of highly dynamic background scenes, the foreground pixels are not contiguous. Therefore, we propose to enforce a post-processing step using Fused Lasso (FL) [47], [54], [56] to strengthen the similarity among the neighboring foreground pixels. FL penalizes the pixels not only by $l_{1}$ norm, but it also preserves the structure of the neighboring pixels. We name this extended algorithm as MSCL-FL for the detection of foreground objects. The proposed MSCL-FL algorithm has shown better performance compared to stateof-the-art methods including TVRPCA [8], 2P-RPCA [15], DP-GMM [17], GRASTA [18], TLSFSD [19], SRPCA [39], LSD [31], 3TD [35], MODSM [36], MLRBS [38], PAWCS [42], SuBSENSE [43], BMTDL [44], GFL [54], GOSUS [55], LR-FSO [56], RMAMR [57], BRTF [63], GoDec [64], and DECOLOR [65] on publicly available datasets, such as Change Detection (CDnet) 2014 [50], I2R [26], Background Models Challenge (BMC) [49], and Wallflower [48].

The remaining content of this paper is organized as follows. In Section II, related work is reviewed. In Section III, we describe our method in detail. The experimental results are discussed in Section IV. Finally, our conclusions and future research directions are presented in Section V.

\section{RELATED WORK}

During the past few years, many research studies have been carried out on background subtraction or foreground detection [8], [11], [17], [20], [25], [42], [65] as well as background initialization [2], [4], [13], [32], [57]. In background subtraction, the emphasis is to improve the accuracy of foreground detection. On the other hand, the task of estimating a foreground-free image is called background modeling. Many surveys have also contributed to these topics [2], [3], [4], [32]. Gaussian Mixture Model (GMM) [45], [60] uses a mixture of Gaussian probability density functions to model color intensity variations at each pixel. Latest GMM enhancements include bidirectional analysis [41] and minimum spanning tree GMM [9]. Most GMM based methods suffer performance degradation in complex dynamic scenes. Recently, deep Convolutional Neural Network (CNN) based methods have also been proposed for foreground segmentation [5], [51], [61]. For instance, Wang et al. [51] proposed a simple and effective $\mathrm{CNN}$ based method for estimating foreground regions. CNN based methods work well in many complex situations however, these methods require significant amount of labelled training data which may not always be available. In contrast, our proposed algorithm is unsupervised therefore do not require labelled training data.

In the current study, we propose a novel algorithm for background modeling, which draws inspiration from subspace learning methods. Wright et al. [53] presented the first proposal of RPCA to handle the outliers in data. Candes et al. [7] used RPCA for background-foreground separation. RPCA-based approaches for background-foreground separation are not ideal for surveillance applications because these approaches suffer from high computational complexity. Moreover, traditional RPCA implementations processed data in batches. Many studies attempting to make the batch methods faster have been reported in literature [8], [64], [65]. However, batch methods are not real-time and mostly work offline. Some online methods have also been reported to handle this problem, while global optimality is still the challenging issue in these approaches [18], [21], [55].

Many authors have contributed interesting studies in the direction of enhancing only foreground detection ${ }^{2}$. For this purpose, a number of constraints have been suggested [8], [21], [31], [54], [65]. For example, Cao et al. [8] improved the performance of foreground detection by proposing the total variation regularized RPCA method. Zhao et al. [65] proposed a markov random field constraint on the foreground matrix to eliminate noise and small background movements. Although the segmentation performance improved, the foreground regions tend to be over-smoothed [31] because of neighboring pixels smoothing constraints.

Unfortunately, research has not been focused in improving low-rank background modeling ${ }^{3}$ [3]. Therefore, there is a need to design a robust algorithm to recover background components in real-life challenging scenarios [32], [37]. We attempt to fill this research gap by proposing a novel notion of encoding spatiotemporal similarity information in the component of the background model. Our idea is motivated by the recently proposed subspace clustering methods in [40], [58], [62]. However, unlike these methods, we incorporate a motion-aware CC and GD based spatiotemporal regularization for clustering low-rank subspace. Our proposed method has some similarity with RMAMR [57] because both methods

\footnotetext{
${ }^{2}$ http://wordpress-jodoin.dmi.usherb.ca/results2014/

${ }^{3}$ http://pione.dinf.usherbrooke.ca/results/
} 


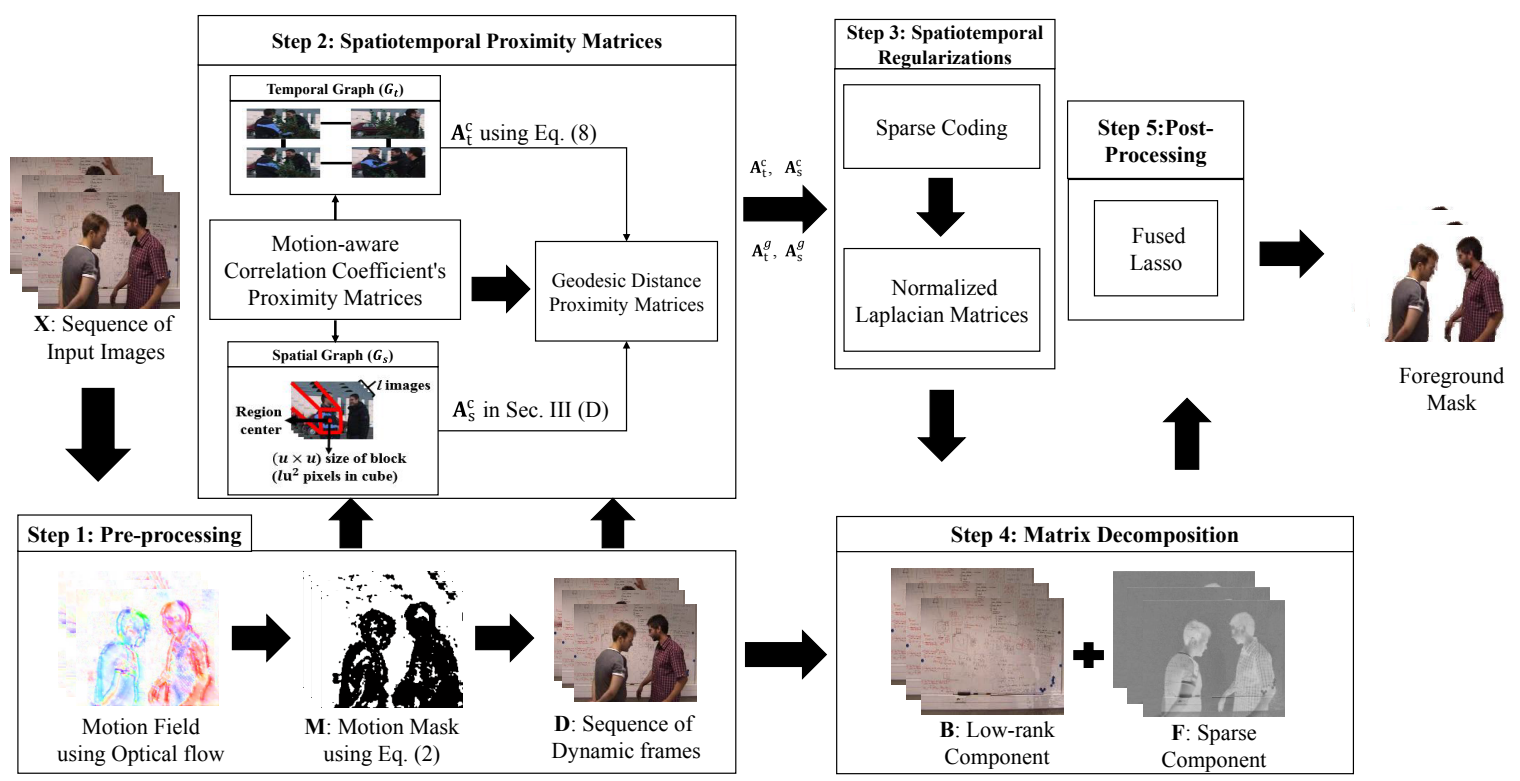

Fig. 4. Schematic illustration of the proposed MSCL algorithm for background estimation and foreground segmentation. Step (1) describes the motion estimation module of sequence $\mathbf{X}$, the creation of motion mask $\mathbf{M}$, and detection of dynamic sequence $\mathbf{D}$. Step (2) describes the construction of spatial and temporal graphs and computation of correlation based and geodesic distance based proximity matrices. In step (3), sparse coding is applied on the proximity matrices computed in step (2) and computation of normalized graph Laplacian matrices. Step (4) shows the decomposition of sequence D into B and F components based on LADMAP optimization. In step (5), the FL constraints are employed on the foreground to ensure continuity.

use motion mask. However, RMAMR has not suggested the spatiotemporal consistency constraints as we proposed using Laplacian matrices of graph structures. Our proposed objective function is novel and to the best of our knowledge, no similar work has been proposed previously.

\section{Proposed Algorithm}

In this section, we describe each step of the proposed MSCL algorithm in detail. The system diagram of the proposed MSCL algorithm is presented in Fig. 4. We first compute the optical flow and create a binary motion mask. This motion mask enables the removal of motionless video frames from the input sequence. Then, spatial and temporal graphs are constructed using the correlation coefficient. The sparse linear decomposition is applied on each graph to estimate the normalized graph Laplacian matrices both in geodesic and correlation space. The four Laplacian matrices computed from these graphs are then incorporated in the RPCA framework. The proposed objective function is solved using the LADMAP optimization scheme. Finally, the Fused Lasso (FL) regularization is applied on the sparse component to enhance the foreground detection.

\section{A. Motion Estimation}

We incorporate motion information in our proposed algorithm by computing the dense optical flow [29] between each pair of consecutive frames in the given sequence $\mathbf{X}$. Using motion information, we compute a motion mask $\mathbf{M}$ and a sequence of dynamic frames D. Let $\mathbf{x}_{i}$ and $\mathbf{x}_{i-1}$ be the two consecutive frames in $\mathbf{X}$ at time $t$ and $t-1$, respectively. Let $v_{i, k}^{x}$ be the horizontal component of the motion vector and $v_{i, k}^{y}$ be the vertical component at position $k$ computed between the frames $\mathbf{x}_{i}$ and $\mathbf{x}_{i-1}$. Let $\mathbf{m}_{i} \in\{0,1\}$ be the corresponding motion mask, which is computed as

$$
m_{i, k}= \begin{cases}1, & \text { if } \sqrt{\left(v_{i, k}^{x}\right)^{2}+\left(v_{i, k}^{y}\right)^{2}}<\tau, \\ 0, & \text { otherwise, }\end{cases}
$$

where $\tau$ is the threshold of motion magnitude, which is computed adaptively as the average of all pixels in the motion field. We select the threshold $\tau$ such that all pixels in $\mathbf{X}$ exhibiting motion larger than $\tau$ definitely belong to the foreground. The threshold $\tau$ is selected to be large enough so that the motion should not result because of noise in the background.

To prepare matrix $\mathbf{D}$ by eradicating the motionless frames in $\mathbf{X}$ using (2), the $i$-th frame in $\mathbf{X}$ is considered to be redundant or motionless if all entries are 1 in the corresponding $i$-th column of $\mathbf{M}$; otherwise, if some entries are 0 , then the frame is considered as dynamic and is appended in matrix $\mathbf{D}$. Using this technique, the dimension of matrix $\mathbf{X}$ may be reduced significantly depending on the number of motionless frames.

\section{B. Mathematical Formulation}

Given the sequence $\mathbf{D} \in \mathbb{R}^{p \times c}$, where $c$ is the number of dynamic frames, we require that the corresponding matrix $\mathbf{B}$ with singular vectors lie in a low-dimensional subspace by minimizing the loss function defined in (1). Here, we define a matrix $\mathbf{M} \in \mathbb{R}^{p \times c 4}$ as a concatenation of $\left[\mathbf{m}_{1}, \mathbf{m}_{2}, . ., \mathbf{m}_{c}\right]$ as computed by (2). We consider the pixel positions in $\mathbf{D}$ corresponding to 0 values in $\mathbf{M}$ as missing data and the remaining pixels as the observed data. The main objective is to estimate these missing values using optimization as:

$$
\min _{\mathbf{B}, \mathbf{F}}|| \mathbf{B}\left\|_{*}+\lambda_{1}\right\| \mathbf{F} \|_{1} \text { such that } \mathbf{M} \circ \mathbf{D}=\mathbf{M} \circ(\mathbf{B}+\mathbf{F}),
$$

\footnotetext{
${ }^{4}$ For simplicity, we use the same notation $\mathbf{M} \in \mathbb{R}^{p \times c}$ for representing the motion mask of only dynamic frames.
} 
where ' $\circ$ ' denotes the element-wise product. The observed $\mathbf{M} \circ \mathbf{D}$ data still contains a significant number of outliers, which actually does not belong to the background. To handle the missing values and outliers effectively in MSCL, we incorporate spatiotemporal smoothness constraints into (3) by encoding the pairwise similarities among the video frames and pixels. We compute a temporal graph across columns of D and a spatial graph across spatial locations of $\mathbf{D}$ using motion-aware Correlation-Coefficient (CC). Based on CCbased adjacency matrix, we compute another representation called Geodesic Distance (GD)-based adjacency matrix.

For each adjacency matrix, we compute a normalized Laplacian matrix, $\mathbf{L}_{t}^{\mathrm{c}} \in \mathbb{R}^{c \times c}$ corresponding to the CC-based adjacency matrix and $\mathbf{L}_{t}^{\mathrm{g}} \in \mathbb{R}^{c \times c}$ corresponding to the GD-based adjacency matrix. Similarly, for the spatial graph, we compute the normalized Laplacian matrices $\mathbf{L}_{s}^{\mathrm{c}} \in \mathbb{R}^{p \times p}$ corresponding to the CC-based adjacency matrix and $\mathbf{L}_{s}^{\mathrm{g}} \in \mathbb{R}^{p \times p}$ corresponding to the GD-based adjacency matrix. The CC-based regularization enforces the background model to be invariant to lighting condition variations and to enhance continuity on linear manifolds. The GD-based regularization ensures the continuity of the background model on the nonlinear manifolds. We observed that the integration of both types of regularization significantly improves the performance of the background model. The proposed MSCL model is then reformulated as

$$
\begin{array}{r}
\min _{\mathbf{B}, \mathbf{F}}\|\mathbf{B}\|_{*}+\Theta_{c}(\mathbf{B}, \mathbf{F})+\Theta_{g}(\mathbf{B}, \mathbf{F})+\lambda_{1}\|\mathbf{F}\|_{1} \\
\text { such that } \mathbf{M} \circ \mathbf{D}=\mathbf{M} \circ(\mathbf{B}+\mathbf{F}),
\end{array}
$$

where the functions $\Theta_{c}$ and $\Theta_{g}$ denote the spatiotemporal CC and GD-based regularization. It is important to note that the functions in (4) are explicitly related with $\mathbf{D}$ and $\mathbf{B}$ and can be shown to be related with $\mathbf{B}$ and $\mathbf{F}$ as: $\Theta_{c}(\mathbf{B}, \mathbf{D})=\Theta_{c}(\mathbf{B}, \mathbf{F}+$ $\mathbf{B})=\Theta_{c}(\mathbf{B}, \mathbf{F})$.

$$
\begin{aligned}
& \Theta_{c}(\mathbf{B}, \mathbf{F})=\frac{\gamma_{1}}{2} \operatorname{Tr}\left(\mathbf{B}^{\top} \mathbf{L}_{s}^{c} \mathbf{B}\right)+\frac{\gamma_{2}}{2} \operatorname{Tr}\left(\mathbf{B} \mathbf{L}_{t}^{c} \mathbf{B}^{\top}\right), \\
& \Theta_{g}(\mathbf{B}, \mathbf{F})=\frac{\gamma_{3}}{2} \operatorname{Tr}\left(\mathbf{B}^{\top} \mathbf{L}_{s}^{g} \mathbf{B}\right)+\frac{\gamma_{4}}{2} \operatorname{Tr}\left(\mathbf{B}^{\top} \mathbf{L}_{t}^{g} \mathbf{B}\right) .
\end{aligned}
$$

We constrain the background model $\mathbf{B}$ to minimize $\operatorname{tr}\left(\mathbf{B}^{\top} \mathbf{L}_{s}^{\mathrm{c}} \mathbf{B}\right), \operatorname{tr}\left(\mathbf{B} \mathbf{L}_{t}^{\mathrm{c}} \mathbf{B}^{\top}\right), \operatorname{tr}\left(\mathbf{B}^{\top} \mathbf{L}_{s}^{\mathrm{g}} \mathbf{B}\right)$, and $\operatorname{tr}\left(\mathbf{B} \mathbf{L}_{t}^{\mathrm{g}} \mathbf{B}^{\top}\right)$, which is a spatiotemporal graph regularization. The parameters $\lambda_{1}, \gamma_{1}, \gamma_{2}, \gamma_{3}, \gamma_{4}>0$ assign relative importance to each of the terms while optimizing (4). If parameters $\gamma_{1}, \gamma_{2}, \gamma_{3}$, and $\gamma_{4}$, are equal to zero, then the model will degenerate to motionaware RPCA.

\section{Temporal Graph Construction}

For the sequence of dynamic frames $\mathbf{D}$, we construct an undirected weighted graph $\mathbf{G}_{t}=\left(\mathbf{V}_{t}, \mathbf{A}_{t}\right)$, such that the vertices $\mathbf{V}_{t}$ correspond to the columns of $\mathbf{D}$ and $\mathbf{A}_{t}$ is the edge weight matrix. First, we compute the pairwise motion-aware correlation coefficient between frames $\left(\mathbf{d}_{i}, \mathbf{d}_{j}\right)[10]$ as

$$
\rho_{t}(i, j)=\frac{\sum_{k=1}^{p} m_{i, j, k}\left(d_{i, k}-\bar{d}_{i}\right)\left(d_{j, k}-\bar{d}_{j}\right)}{\sqrt{\sum_{k=1}^{p} m_{i, j, k}\left[\left(d_{i, k}-\bar{d}_{i}\right)^{2}+\left(d_{j, k}-\bar{d}_{j}\right)^{2}\right]}},
$$

where $k$ is the pixel position and $m_{i, j, k}=m_{i, k} \wedge m_{j, k}$ is a motion indicator computed from the motion masks of the two frames, $m_{i, j, k}=1$ if both $m_{i, k}=1$ and $m_{j, k}=1$; otherwise, it will be zero. $\bar{d}_{i}$ is the mean of the frame $\mathbf{d}_{i}$. The value of $\rho_{t}(i, j)$ ranges from -1 to +1 , where +1 indicates a perfect linear relationship and -1 is a perfect inverse relationship, which may happen because of abrupt light intensity variations. We compute the weight matrix $\mathbf{A}_{t}$ as normalized Euclidean distance matrix:

$$
A_{t}(i, j)=\sqrt{2\left(1-\rho_{t}(i, j)\right)} .
$$

The proximity matrix $\mathbf{A}_{t}^{c}$ of the graph $\mathbf{G}_{t}$ is computed as

$$
\mathbf{A}_{t}^{c}=\exp \left(-\mathbf{A}_{t} / 2 \sigma_{t}^{2}\right)
$$

where $\sigma_{t}^{2}$ is the smoothing factor that strengthens the fusion between the adjacent pixels. We use $\sigma_{t}$ as an average distance among the nodes in $\mathbf{G}_{t}$ [40]. If $A_{t}^{\mathrm{c}}(i, j)>0$, then there is an edge between nodes $\mathbf{d}_{i}$ and $\mathbf{d}_{j}$ in $\mathbf{G}_{t}$.

\section{Spatial Graph Construction}

Using motion-aware correlation coefficient, we compute a spatial graph $\mathbf{G}_{s}=\left(\mathbf{V}_{s}, \mathbf{A}_{s}\right)$ such that $\mathbf{V}_{s}$ corresponds to the rows of matrix $\mathbf{D}$ and $\mathbf{A}_{s}$ is the edge weight matrix computed similar to $\mathbf{A}_{t}$. The spatial graph $\mathbf{G}_{s}$ complements the information captured by the temporal graph $\mathbf{G}_{t}$. Given that $\mathbf{G}_{s}$ encodes the notion of similarity among the spatial locations; therefore, it will enforce smoothness on the patch level in spatial dimensions in the low-rank model. Moreover, computing the correlation coefficient among the rows of patches allows the use of local information in the image sequence.

For each pixel in the first frame in $\mathbf{D}$, we consider a patch of size $u \times u$ pixels in the corresponding 2-D image. Therefore, for each row of pixels in $\mathbf{D}$, we obtain a row of patches or patch-row having the size $c \times u \times u$. The motion-aware correlation coefficient $\rho_{s}(i, j)$ is computed between all pairs of patch-rows resulting in a $p \times p$ matrix of correlations, which is transformed to normalized Euclidean distance $\mathbf{A}_{s}$ by using $A_{s}(i, j)=\sqrt{2\left(1-\rho_{s}(i, j)\right)}$. Spatial proximity matrix $\mathbf{A}_{s}^{c}$ is obtained by $A_{s}^{c}(i, j)=\exp \left(-A_{s}(i, j) / 2 \sigma_{s}^{2}\right)$, where $\sigma_{s}^{2}$ acts as a spatial smoothing factor computed as an average distance among nodes in $\mathbf{G}_{s}$.

\section{E. Geodesic Distance Based Proximity Matrices}

For the spatial and temporal graphs $\mathbf{G}_{t}=\left(\mathbf{V}_{t}, \mathbf{A}_{t}\right)$ and $\mathbf{G}_{s}=\left(\mathbf{V}_{s}, \mathbf{A}_{s}\right)$, we compute all pairs of shortest distance or Geodesic Distance (GD) [22], [23]. We obtain two more proximity matrices $\mathbf{A}_{t}^{g} \in \mathbb{R}^{c \times c}$ and $\mathbf{A}_{s}^{g} \in \mathbb{R}^{p \times p}$ such that the $i$-th column in these matrices corresponds to the geodesic distances of the $i$-th node from the rest of the nodes in that graph. These matrices will be used to construct regularization, which makes the low-rank robust to the nonlinear variations in spatial and temporal dimensions.

For the temporal graph $\mathbf{G}_{t}$, the geodesic distance matrix $\mathbf{P}_{t}^{g}$ is computed using $\mathbf{A}_{t}$. For the spatial graph $\mathbf{G}_{s}$, the geodesic distance matrix $\mathbf{P}_{s}^{g}$ is computed using $\mathbf{A}_{s}$. The geodesic distance between two nodes is more meaningful than the Euclidean distance computed in the image space because two frames may have smaller Euclidean distance, while geodesic 
distance is larger along the nonlinear manifold. Using the geodesic distance matrix $\mathbf{P}^{g} \in\left\{\mathbf{P}_{t}^{g}, \mathbf{P}_{s}^{g}\right\}$, we compute the geodesic proximity matrices $\mathbf{A}^{g} \in\left\{\mathbf{A}_{t}^{g}, \mathbf{A}_{s}^{g}\right\}$,

$$
\mathbf{A}^{g}=\exp \left(-\mathbf{P}^{g} \circ \mathbf{P}^{g} / 2 \sigma_{g}^{2}\right),
$$

where $\sigma_{g} \in\left\{\sigma_{g}^{s}, \sigma_{g}^{t}\right\}$ provides smoothing in the geodesic space and computed as the average distance between the nodes in the corresponding graphs. In general, if a node $\mathbf{d}_{i}$ is not reachable from node $\mathbf{d}_{j}$, then $A^{g}(i, j)=\infty$ and $P^{g}(i, j)=0$.

\section{F. Sparse Linear Coefficients Proximity Matrices}

We observe that the columns of a proximity matrix $\mathbf{A} \in$ $\left\{\mathbf{A}_{t}^{c}, \mathbf{A}_{s}^{c}, \mathbf{A}_{t}^{g}, \mathbf{A}_{s}^{g}\right\}$ span low dimensional subspaces compared to the overall matrix dimensions because the size of the temporal proximity matrices $\mathbf{A}_{t}^{c}$ and $\mathbf{A}_{t}^{g}$ is the number of frames $\times$ number of frames. In case there is no change in the background, ideally, the rank of both of these matrices will be 1. However, given the dynamic nature of the background, we observe that the rank of these matrices will be larger than 1, but much smaller than the number of frames. In Fig. 3 , we plot the temporal and spatial graphs of the dynamic background sequences. Each graph contains multiple nonlinear clusters showing the presence of multiple manifolds. Therefore, a better background model can be computed by using subspace-based spectral clustering [12]. We compute the proximity matrices by applying sparse linear decomposition on the matrix A. The computation of normalized Laplacian using sparse linear coefficients has been proposed in subspace sparse spectral clustering [12], which may be reviewed for more theoretical details.

Let $\mathbf{a}_{i}$ be the $i$-th column of $\mathbf{A}$, which is decomposed as a linear combination of the rest of the matrix $\hat{\mathbf{A}}=\mathbf{A} \backslash \mathbf{a}_{i}$. $\hat{\mathbf{A}}$ is the same as $\mathbf{A}$ except that the $\mathbf{a}_{i}$ column is replaced by a column of zeros. We find an optimal solution for sparse linear coefficients $\boldsymbol{\alpha}_{i}$ :

$$
\boldsymbol{\alpha}_{i}^{*}:=\arg \min _{\boldsymbol{\alpha}_{i}}\left(\left\|\mathbf{a}_{i}-\hat{\mathbf{A}} \boldsymbol{\alpha}_{i}\right\|_{2}^{2}+\lambda_{2}\left\|\boldsymbol{\alpha}_{i}\right\|_{1}\right),
$$

where $\lambda_{2}$ gives a relative importance to the $\ell_{1}$ norm factor, which approximates the $\ell_{o}$ norm actually required for sparsity. $\lambda_{2}$ is automatically computed from the data [12]. The sparse linear decomposition with $l_{1}$-norm constraints given by (10) is an unconstrained convex optimization problem also known as the Least Absolute Selection and Shrinkage Operator (LASSO) [46]. We solve this problem using a fast solution available in the Least Angle Regression (LARS) framework [12], which has the same asymptotic complexity as the simple least squares regression.

Sparse linear decomposition is applied on each column of $\mathbf{A}$ and the resulting $\boldsymbol{\alpha}_{i}$ are arranged as columns resulting in a matrix of sparse linear coefficients $\mathbf{E}$ where $\mathbf{E} \in$ $\left\{\mathbf{E}_{t}^{c}, \mathbf{E}_{s}^{c}, \mathbf{E}_{t}^{g}, \mathbf{E}_{s}^{g}\right\}$.

\section{G. Computing Normalized Laplacian Matrices}

The matrices of linear coefficients may not be symmetric $\mathbf{E} \neq \mathbf{E}^{\top}$, which is partially because the set of vectors used to represent each vector $\mathbf{a}_{i} \in \mathbf{A}$ is slightly different from the set used to represent $\mathbf{a}_{j} \in \mathbf{A}$. We make this relationship normalized and symmetric by taking the average of both coefficients normalized by the maximum value of its own set [12]:

$$
E(i, j)=E(j, i)=\frac{1}{2}\left(\left|\frac{\alpha_{i, j}}{\max \left(\boldsymbol{\alpha}_{i}\right)}\right|+\left|\frac{\alpha_{j, i}}{\max \left(\boldsymbol{\alpha}_{j}\right)}\right|\right) .
$$

Using each normalized symmetric matrix $\mathbf{E}$ of sparse linear coefficients, we compute a normalized graph Laplacian matrix $\mathbf{L}$ where $\mathbf{L} \in\left\{\mathbf{L}_{t}^{c}, \mathbf{L}_{s}^{c}, \mathbf{L}_{t}^{g}, \mathbf{L}_{s}^{g}\right\}$

$$
\mathbf{L}=\mathbf{I}-\mathbf{W}^{-1 / 2} \mathbf{E} \mathbf{W}^{-1 / 2},
$$

where $\mathbf{I}$ is an identity matrix of the same size as $\mathbf{E}, \mathbf{W}$ is the degree matrix whose diagonal entries are given by $W(j, j)=$ $\sum_{i} E(i, j)$, and non-diagonal values are zero. The normalized Laplacian matrices are used to constrain the background model $\mathbf{B}$, minimizing either the trace of $\mathbf{B}^{\top} \mathbf{L} \mathbf{B}$ or $\mathbf{B L B}{ }^{\top}$ depending on the dimensions of $\mathbf{L}$, as shown in the objective function (4). In the following section, we present the solution of the proposed objective function.

\section{H. Proposed LADMAP Optimization}

Model (4) is essentially a convex optimization problem, which is solved using the Linearized Alternating Direction Method with Adaptive Penalty (LADMAP) [27], [28], [52]. For this purpose, the linear equality constraints in (4) are removed by employing following augmented Lagrangian formulation:

$$
\begin{aligned}
\mathcal{L}(\mathbf{B}, \mathbf{F}, \mathbf{Y}, \mu)= & \min _{\mathbf{B}, \mathbf{F}}\|\mathbf{B}\|_{*}+\Theta_{c}(\mathbf{B}, \mathbf{F})+\Theta_{g}(\mathbf{B}, \mathbf{F}) \\
& +\lambda_{1}\|\mathbf{F}\|_{1}+\operatorname{tr}(\mathbf{Y}(\mathbf{M} \circ(\mathbf{D}-\mathbf{B}-\mathbf{F}))) \\
& +\frac{\mu}{2}\|\mathbf{M} \circ(\mathbf{D}-\mathbf{B}-\mathbf{F})\|_{F}^{2},
\end{aligned}
$$

where $\mathbf{Y} \in \mathbb{R}^{p \times c}$ is a Lagrangian multiplier matrix and $\mu>0$ controls the penalty for violating the linear constraints. Optimizing directly the primary variables $\mathbf{B}, \mathbf{F}$, and $\mathbf{Y}$, the LADMAP method solves each variable iteratively one after another. LADMAP uses less auxiliary variables without matrix inversions and converges faster than the original ADM [52]. We derive the solutions to use the proximity operator of the nuclear norm effectively and $l_{1}$-norm in solving subproblems including Laplacian terms.

Updating B: According to LADMAP, the linearization only over the augmented quadratic penalty term in (12) solves subproblems $\mathbf{B}_{k+1}$ and $\mathbf{F}_{k+1}$ (in this case, $k$ is the iteration index). By fixing $\mathbf{F}$, the update for background $\mathbf{B}_{k+1}$ at the $(k+1)$-th iteration is

$$
\begin{aligned}
\mathbf{B}_{k+1}= & \arg \min _{\mathbf{B}} \mathcal{L}\left(\mathbf{B}, \mathbf{F}_{k}, \mathbf{Y}_{k}, \mu_{k}\right)=\arg \min _{\mathbf{B}}\|\mathbf{B}\|_{*}+\Theta_{c}(\mathbf{B}, \mathbf{F}) \\
& +\Theta_{g}(\mathbf{B}, \mathbf{F})+\operatorname{Tr}\left(\mathbf{Y}_{k}\left(\mathbf{M} \circ\left(\mathbf{D}-\mathbf{B}-\mathbf{F}_{k}\right)\right)\right) \\
& +\frac{\mu_{k}}{2}\left\|\mathbf{M} \circ\left(\mathbf{D}-\mathbf{B}-\mathbf{F}_{k}\right)\right\|_{F}^{2}
\end{aligned}
$$

If $\gamma_{1}, \gamma_{2}, \gamma_{3}, \gamma_{4}>0$ in functions $\Theta_{c}$ and $\Theta_{g}$, then optimizing subproblem $\mathbf{B}_{k+1}$ does not lead to the exact solution. Therefore, the convergence analysis provided in [28] is not applicable. To use the closed-form solution to the proximity operator of the nuclear norm, which is given by Singular Value Thresholding (SVT) operator [6], we further linearize the 
graph regularization terms to simplify the subproblem $\mathbf{B}_{k+1}$. According to Lemma 2.1 in [1], the trace norm of any graph regularization terms, e.g., $\frac{\gamma_{1}}{2} \operatorname{tr}\left(\mathbf{B}^{\top} \mathbf{L}_{s}^{\mathrm{c}} \mathbf{B}\right)$ in (12) can be upper bounded by its proximal approximation which describes the local linearization of $\frac{\gamma_{1}}{2} \operatorname{tr}\left(\mathbf{B}^{\top} \mathbf{L}_{s}^{\mathrm{c}} \mathbf{B}\right)$ at $\mathbf{B}_{k}$. Then, the solution of (14) leads to the following problem after applying simple linear algebra as

$$
\mathbf{B}_{k+1}=\underset{\mathbf{B}}{\operatorname{argmin}}\|\mathbf{B}\|_{*}+\frac{\eta_{1}}{2} \times\left\|\mathbf{M} \circ\left(\mathbf{B}-\mathbf{B}_{k}\right)+\frac{\mathbf{H}}{\eta_{1}}\right\|_{F}^{2}
$$

For our convenience, we denote

$$
\left\{\begin{array}{l}
\eta_{1}=\mu_{k}+\rho_{1}, \\
\rho_{1}=\gamma_{1}\left\|\mathbf{L}_{s}^{\mathrm{c}}\right\|+\gamma_{2}\left\|\mathbf{L}_{t}^{\mathrm{c}}\right\|+\gamma_{3}\left\|\mathbf{L}_{s}^{\mathrm{g}}\right\|+\gamma_{4}\left\|\mathbf{L}_{t}^{\mathrm{g}}\right\| \\
\mathbf{H}=-(\mathbf{M} \circ \mathbf{D}) \tilde{\mathbf{Y}}_{k}+\boldsymbol{\rho}_{2}, \\
\boldsymbol{\rho}_{2}=\gamma_{2} \mathbf{B}_{k} \mathbf{L}_{t}^{\mathrm{c}}+\gamma_{4} \mathbf{B}_{k} \mathbf{L}_{t}^{\mathrm{g}}+\left[\gamma_{1} \mathbf{B}_{k}^{\top} \mathbf{L}_{s}^{\mathrm{c}}+\gamma_{3} \mathbf{B}_{k}^{\top} \mathbf{L}_{s}^{\mathrm{g}}\right]^{\top}, \\
\tilde{\mathbf{B}}_{k}=\left(\mathbf{M} \circ \mathbf{B}_{k}\right)-\frac{1}{\eta_{1}}\left[-(\mathbf{M} \circ \mathbf{D}) \tilde{\mathbf{Y}}_{k}+\boldsymbol{\rho}_{2}\right],
\end{array}\right.
$$

To solve $\mathbf{B}_{k+1}$, first we need to compute the parameter $\mu_{k}$ and matrix $\tilde{\mathbf{Y}}_{k}$ as

$$
\left\{\begin{array}{l}
\mu_{k+1}=\mu_{k}+\rho_{0} \mu_{\max } \\
\mu_{\max }=\frac{\max \left\{\gamma_{2}\left\|\mathbf{L}_{t}^{c}\right\|+\gamma_{4}\left\|\mathbf{L}_{t}^{g}\right\|, \gamma_{1}\left\|\mathbf{L}_{s}^{c}\right\|+\gamma_{3}\left\|\mathbf{L}_{s}^{g}\right\|\right\}}{\|\mathbf{M} \circ \mathbf{D}\|^{2}-\lambda_{1}} \\
\tilde{\mathbf{Y}}_{k}=\mathbf{Y}_{k}+\mu_{k} \mathbf{M} \circ\left(\mathbf{D}-\mathbf{B}_{k}-\mathbf{F}_{k}\right), \\
\mathbf{Y}_{k+1}=\mathbf{Y}_{k}+\mu_{k} \mathbf{M} \circ\left(\mathbf{D}-\mathbf{B}_{k+1}-\mathbf{F}_{k+1}\right)
\end{array}\right.
$$

where $\rho_{0}$ is constant and the regularization e.g., $\left\|\mathbf{L}_{s}^{\mathrm{c}}\right\|$ is known as the spectral norm of a matrix $\mathbf{L}_{s}^{\mathrm{c}}$, which is the largest singular value of matrix $\mathbf{L}_{s}^{\mathrm{c}}$. The $\mathbf{B}_{k+1}$ has the following closed-form solution as

$$
\mathbf{B}_{k+1}=\mathbf{U T}_{\tau}(\boldsymbol{\Sigma}) \mathbf{V}^{\top}, \text { where }\left(\mathbf{U}, \boldsymbol{\Sigma}, \mathbf{V}^{\top}\right)=\operatorname{SVD}\left(\tilde{\mathbf{B}}_{k}\right)
$$

where $\boldsymbol{\Sigma}$ is the singular value matrix of $\tilde{\mathbf{B}}_{k}$. The operator $\mathrm{T}_{\tau}(\cdot)$ is the SVT [6], which is defined by element-wise $\tau$ thresholding of $\boldsymbol{\Sigma}$. Especially, let $\sigma_{i}$ be the $i$-th diagonal element of $\boldsymbol{\Sigma}$, then $\mathrm{T}_{\tau}(\boldsymbol{\Sigma})$ is a diagonal matrix defined by $\mathrm{T}_{\tau}(\boldsymbol{\Sigma})=\operatorname{diag}\left(\left\{\left(\sigma_{i}\right)-\tau\right\}_{+}\right)$, where $a_{+}$is the positive part of $a_{+}=\max (0, a)$.

Updating F: When $\mathbf{B}$ is fixed, to update $\mathbf{F}_{k+1}$, we derive from (13) the following sub-problem:

$$
\begin{aligned}
\mathbf{F}_{k+1}= & \arg \min _{\mathbf{F}} \mathcal{L}\left(\mathbf{B}_{k+1}, \mathbf{F}, \mathbf{Y}_{k}, \mu_{k}\right) \\
& =\arg \min _{\mathbf{F}} \lambda\|\mathbf{F}\|_{1}+\operatorname{Tr}\left(\mathbf{Y}\left(\mathbf{M} \circ\left(\mathbf{D}-\mathbf{B}_{k+1}-\mathbf{F}\right)\right)\right) \\
& +\frac{\mu_{k}}{2}\left\|\mathbf{M} \circ\left(\mathbf{D}-\mathbf{B}_{k+1}-\mathbf{F}\right)\right\|_{F}^{2}
\end{aligned}
$$

The closed-form solution of the above problem is

$$
\mathbf{F}_{k+1}=\mathbf{S}_{\lambda / \mu_{k}}\left(\mathbf{M} \circ\left(\mathbf{D}-\mathbf{B}_{k+1}\right)+\frac{\tilde{\mathbf{Y}}_{k}}{\mu_{k}}\right)
$$

where $S_{\tau}(\cdot)$ is the shrinkage operation [27] defined by

$$
\mathbf{S}_{\tau}(\mathbf{F})=\operatorname{sgn}(\mathbf{F}) \circ \max \left\{|\mathbf{F}|-\tau_{1}, 0\right\} .
$$

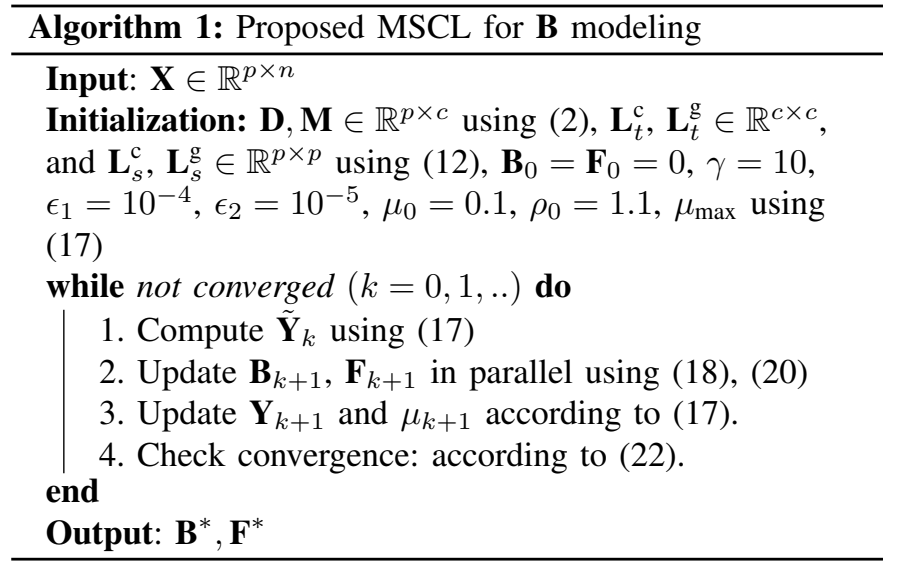

Convergence Condition: According to the KKT condition, the following criterion is defined for the sub-optimality of the solution of problem (4)

$$
\begin{aligned}
& \left\|\mathbf{M} \circ\left(\mathbf{D}-\mathbf{B}_{k+1}-\mathbf{F}_{k+1}\right)\right\| /\|\mathbf{M} \circ \mathbf{D}\|<\epsilon_{1} \text { and } \\
& \max \left\{\rho_{3}, \rho_{4}\right\} \leq \epsilon_{2} \text {, where } \rho_{3}=\mu_{k}\left\|\mathbf{M} \circ\left(\mathbf{F}_{k+1}-\mathbf{F}_{k}\right)\right\|, \\
& \rho_{4}=\frac{\mu_{k}+2 \rho_{1}}{\sqrt{\lambda_{1}}}\left\|\mathbf{M} \circ\left(\mathbf{B}_{k+1}-\mathbf{B}_{k}\right)\right\|,
\end{aligned}
$$

where $\epsilon_{1}$ and $\epsilon_{2}$ are the appropriate tolerances. Based on the stopping criteria defined in (22), the sequences $(\mathbf{B}, \mathbf{F}, \mathbf{Y})$ generated by the revised LADMAP converges to an optimal solution of problem (4). Algo. 1 describes the summary of MSCL.

\section{Foreground Detection}

The additional constraints on the low-rank matrix enhanced the quality of the background model and improved the foreground detection. Often, the foreground is relatively smaller and sparser compared to the background. Consequently, minimizing the foreground term by imposing the $l_{1}$ norm on $\mathbf{F}$ makes the estimation even more sparser. We observe that without considering the spatial connectivity among the adjacent foreground pixels, especially in dynamic backgrounds, it is difficult to obtain precise foreground segmentation (Fig.3).

To improve the foreground segmentation, we constrain the foreground pixels by employing Fused Lasso (FL) [47] as a post-processing step. We name the resulting algorithm as MSCL-FL for foreground detection, which is robust against dynamic background scenes. In MSCL-FL, it is assumed that if a pixel belongs to the foreground, then its neighbors would also belong to the foreground. The overall goal of MSCL-FL is to minimize the following energy function as:

$$
\|\mathbf{F}\|_{\mathrm{FL}}=\sum_{k=1}^{c}\left\{\left\|\mathbf{f}^{(k)}\right\|_{1}+\beta \sum_{(i, j) \in N} w_{i j}^{(k)}\left|f_{i}^{(k)}-f_{j}^{(k)}\right|\right\},
$$

where $\mathbf{f}^{(k)}$ is the $k$-th vector of $\mathbf{F}$ and $N$ is the spatial neighborhood system, i.e., $(i, j) \in N$ when both pixels $i$ and $j$ are spatially connected. The first term $\|\mathbf{f}\|_{1}$ is the observed data term that connects each pixel to background and foreground nodes. The second term is known as the smoothness term, which represents the relationship between 


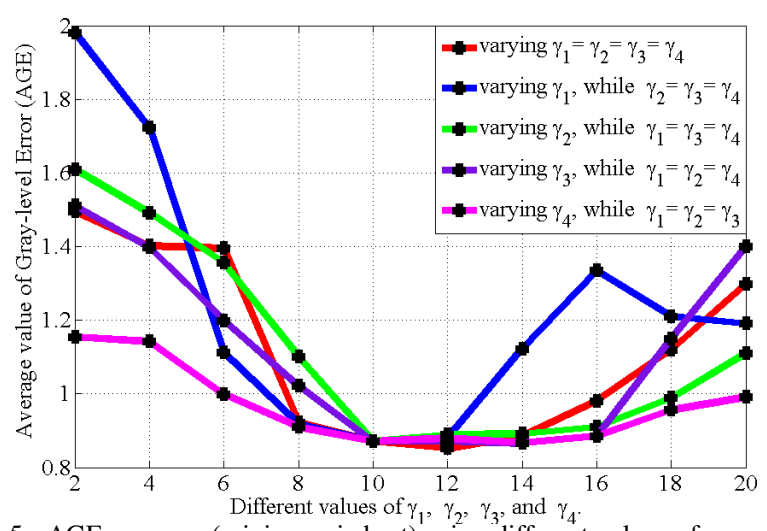

Fig. 5. AGE measure (minimum is best) using different values of parameters $\gamma_{1}, \gamma_{2}, \gamma_{3}$, and $\gamma_{4}$. An experiment performed on "CameraParameter" sequence of the SBM.net dataset [37].

adjacent pixels $i$ and $j . \beta$ is a scalar parameter and $w_{i j}$ is the adaptive weighting factor that stabilizes the fusion between pixels as $w_{i j}^{(k)}=\exp -\left\|d_{i}^{(k)}-d_{j}^{(k)}\right\|_{2}^{2} / 2 \sigma^{2}$, where $d$ is the pixel intensity of $k$-th frame and $\sigma$ is the spread of the Gaussian function. The smoothness term encourages the same labeling to similar neighboring pixels. The energy function (23), which is nonconvex, is difficult to solve especially if the dimensionality is large. Therefore, we use the fast parametric flow method [14], [54].

\section{EXPERIMENTS}

We performed extensive experiments on five publicly available video datasets including Scene background modeling.net (SBM.net) [37], Change Detection.net (CDnet) 2014 [50], Background Models Challenge (BMC) [49], I2R [26], and Wallflower [48]. The results are compared with 23 state-ofthe-art methods, such as TVRPCA [8], RMR [11], 2P-RPCA [15], RFSA [16], DP-GMM [17], GRASTA [18], TLSFSD [19], our previous algorithm MAGRPCA [20], LSD [31], 3TD [35], MODSM [36], MLRBS [38], SRPCA [39], PAWCS [42], SuBSENSE [43], BMTDL [44], GFL [54], GOSUS [55], LR-FSO [56], RMAMR [57], BRTF [63], GoDec [64], and DECOLOR [65], using original author implementations. Background models are compared using Average Gray-level Error (AGE), percentage of Error Pixels (pEPs), Percentage of Clustered Error Pixels (pCEPs), Multi Scale Structural Similarity Index (MSSSIM), Color image Quality Measure (CQM), and PSNR [32], [37]. We aim to minimize AGE, pEPs, and pCEPs for more accurate background model recovery while maximizing MSSSIM, PSNR, and CQM.

The solution of the proposed model (4) requires parameters $\lambda_{1}, \gamma_{1}, \gamma_{2}, \gamma_{3}$, and $\gamma_{4}$. We use $\lambda_{1}=1 / \sqrt{\max (p, c)}$, where $p$ is the number of pixels and $c$ is the number of dynamic frames in matrix D [7]. In our experiments, we used $\gamma_{1}=\gamma_{2}=\gamma_{3}=$ $\gamma_{4}=10$. We empirically verified that further tuning of these parameters may yield some improvement in accuracy (Fig. 5). However, to make the experiments repeatable, all results are reported with the same value given previously. The parameter $\mu_{k}$ in (12) is initialized with 0.1 and then adjusted adaptively in the later iterations. For the construction of $\mathbf{G}_{s}$ on image patches, we used the patch size of $5 \times 5$ pixels. The parameters
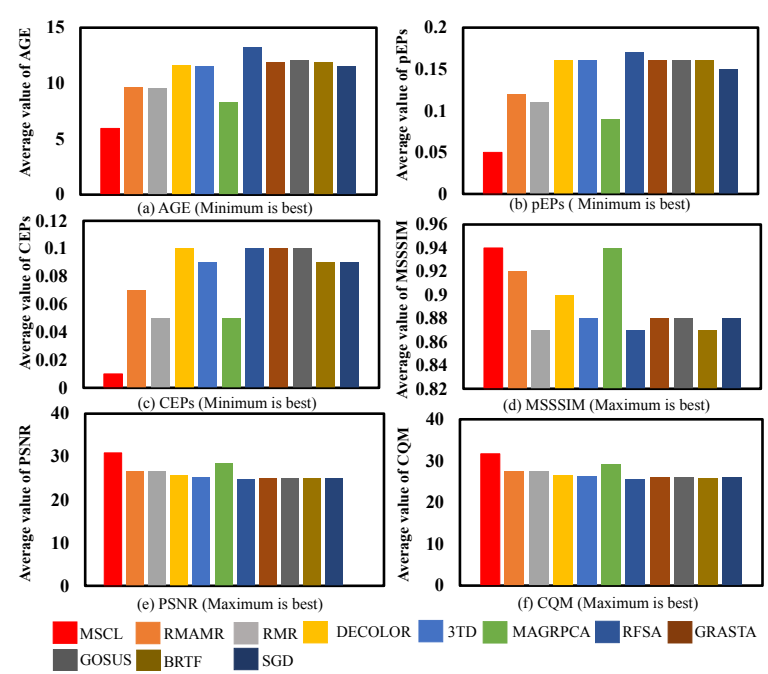

Fig. 7. Average performance on each metric of MSCL with existing methods on the overall SBM.net dataset.

required by the LADMAP are used as recommended by the original authors [28], [30], [58]. In addition, we used the PROPACK library [24] for LADMAP implementation.

\section{A. Evaluation of MSCL on SBMI Dataset}

We evaluated our proposed MSCL algorithm on the SBM.net dataset, which contains 8 challenging categories and 80 videos (Fig. 6). In contrast to the existing datasets, the SBM.net comprises complex scenes in which no foregroundfree images are available and the background is largely occluded by foreground objects. More details can be found at http://scenebackgroundmodeling.net. On the average, our algorithm outperformed all existing methods in terms of 6 accuracy measures included in the study [37] (Figs. 7 and 8). The MSCL outperformed all existing methods in three categories, namely, 'Basic', 'Clutter', and 'Illumination changes' (Fig. 8). A visual comparison of the estimated background model with 10 best performing methods over 8 selected sequences (one sequence per category) is illustrated in Fig. 6.

In Fig.8, we present only 5 noteworthy methods and analyze their average performance on each category using the AGE metric, which is the $l_{1}$-norm of the difference of ground truth and the estimated background image. Lower AGE value denotes better background estimation. For the 'Basic' category, only MSCL and RMR produced good results, while the remaining methods exhibit some discrepancies in the form of outliers because background pixels are mostly visible all the time in these sequences, although there are no frames containing complete background. In the challenging sequences of 'Clutter' category, the background remains largely occluded by the foreground objects. Fig. 8 shows that the proposed algorithm attains, on the average, the best accuracy in terms of AGE value. In the compared methods, overwhelming outliers of foreground objects were incorporated into the estimated background model (Fig. 6, $3^{\text {rd }}$ row).

For 'Jitter' sequences, most of the methods perform good estimation of the background component. In this case, the proposed MSCL algorithm has shown the best performance. RMAMR and MAGRPCA also use motion information; there- 


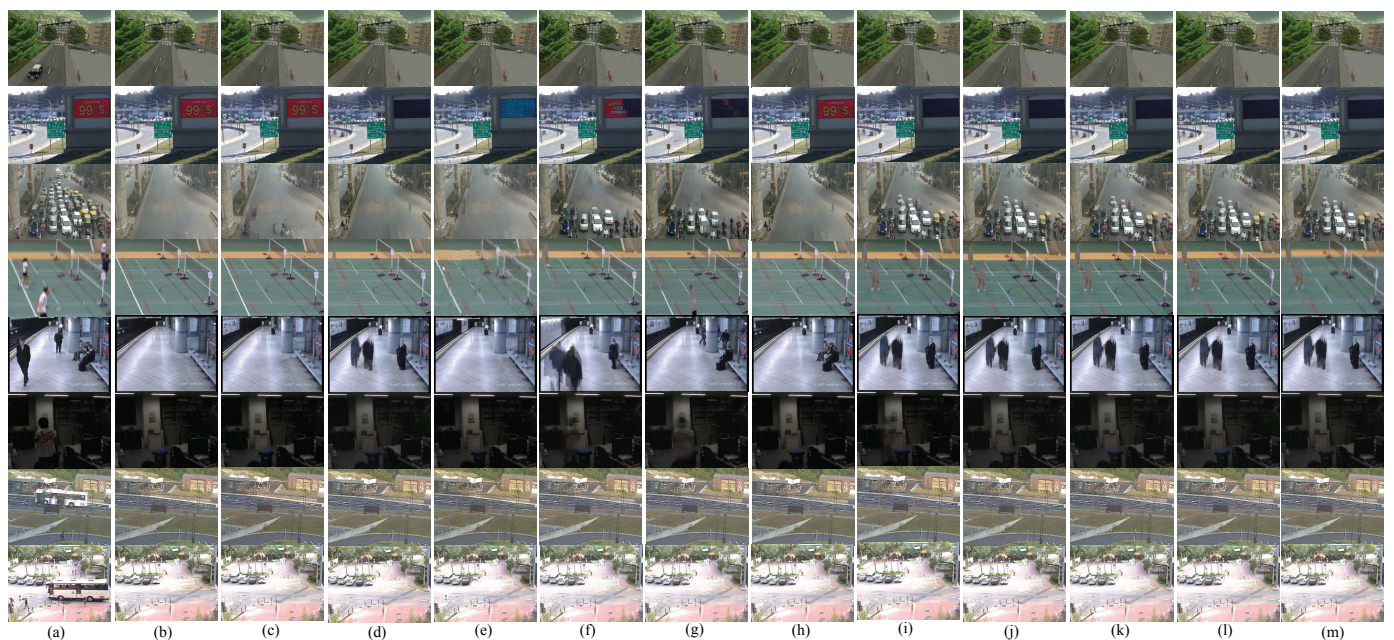

Fig. 6. Qualitative results of the proposed method. (a) 8 images from the input sequences, (b) ground truth images, (c) estimated background model by the proposed MSCL, (d) RMAMR, (e) RMR, (f) DECOLOR, (g) 3TD, (h) MAGRPCA, (i) RFSA, (j) GRASTA, (k) GOSUS, (l) BRTF, and (m) GoDec. From top to bottom: each input sequence is selected from each category. (1)sequence '511' from 'Basic', (2) 'advertisementBoard' from 'Background Motion', (3) 'boulevardJam' from 'Clutter', (4) 'badminton' from 'Jitter', (5) 'AVSS2007' from 'Intermittent Motion', (6) 'CameraParameter' from 'Illumination Changes', (7) 'BusStopMorning' from 'Very Long', and (8) 'CUHK Square' from 'Very Short'.

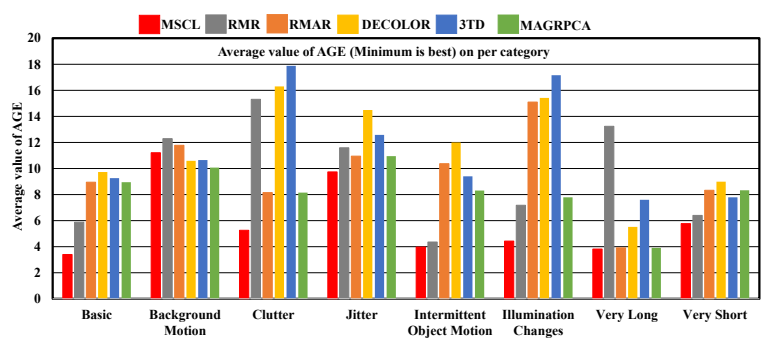

Fig. 8. Performance of each method according to each category.

fore, these methods also show better performance. For complex dynamics as presented by the sequences in the 'Background Motion' category, all methods have shown relatively poor performance because of the sudden change in the background in the presence of foreground objects, which always remain visible. In this category, MSCL is in the middle of the spectrum obtaining an AGE score of 11.21, while the best performing competitor DECOLOR obtained 10.5. Note that the previous version of our proposed algorithm MAGCPCA has obtained the best accuracy at 10.07, which shows that the inclusion of the correlation coefficient-based regularization has caused some degradation in this case, while the overall accuracy has significantly increased.

In the 'Intermittent Motion' category, some foreground objects remain motionless and then start moving, creating outlier in the background. Our algorithm considered the motionless frames as redundant and removed them, which helped in reducing the outliers. This step is only useful for this category, while in other cases, this step has no effect. Fig. 8 demonstrates that majority of the methods failed to cope with incorporated outliers because of motionless foreground objects. Only MSCL and RMR methods effectively handled these outliers in the final estimation of background. The proposed MSCL provides the best performance in terms of AGE measure. For sequences that belong to the 'Illumination Changes' category, Fig. 8 shows that the proposed algorithm has exhibited significant improvement over current methods, which have shown degraded performance. The performance of the proposed algorithm is improved because of the use of the correlation coefficient for the computation of adjacency matrices, which are robust to illumination variations. All of the compared methods have shown relatively good performance on 'Very Long' and 'Very Short' categories.

Considering all categories of the SBM.net dataset, on the average, the MSCL obtained an AGE score of 5.95 and has been ranked as the best performer (http://scenebackgroundmodeling.net). The improved performance is attributed to the spatiotemporal regularization, which was able to handle the outliers effectively and ensured smoothness of the background model on temporal and spatial manifolds.

\section{B. Importance of Different Regularizations}

To find the relative contribution of different regularizations in (4), we conduct a series of experiments. Setting all $\gamma$ 's $=0$ results in LADMAP based RPCA, $\gamma_{2}=\gamma_{4}=0$ results in spatially regularized RPCA, $\gamma_{1}=\gamma_{3}=0$ results in temporally regularized RPCA, $\gamma_{1}=\gamma_{2}=0$ results in nonlinear spatiotemporal regularized RPCA, and $\gamma_{3}=\gamma_{4}=0$ results in linear spatiotemporal regularized RPCA. In addition, we also implemented the proposed objective function without using the Sparse Subspace Clustering (SSC) [12]. Fig. 9 shows the comparison of these variants with the proposed algorithm with the full objective function on six different sequences from the SBM.net dataset. In all sequences, the performance of RPCA has remained the least (shown in black) and the performance of the proposed MSCL has remained the best (shown in red). The performance of MSCL without SSC (shown in green) has remained poor compared to the proposed MSCL with only spatial, only temporal, and both spatiotemporal constraints.

In the case of the cluttered scene (Fig.9(a)), the temporal regularization (shown in blue) has performed better compared to spatial regularization (shown in magenta) because of the presence of more outliers along the rows of matrix $\mathbf{D}$. In 


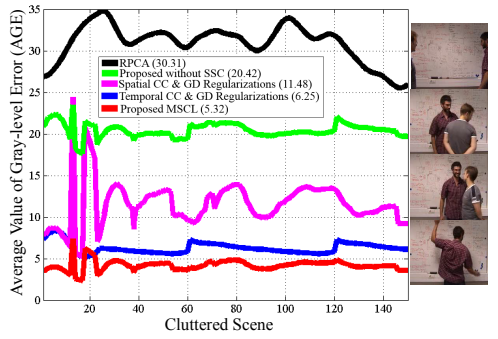

(a)

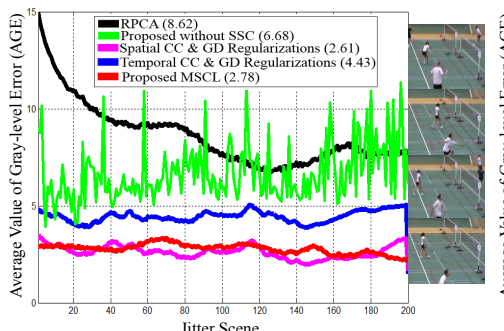

(d)

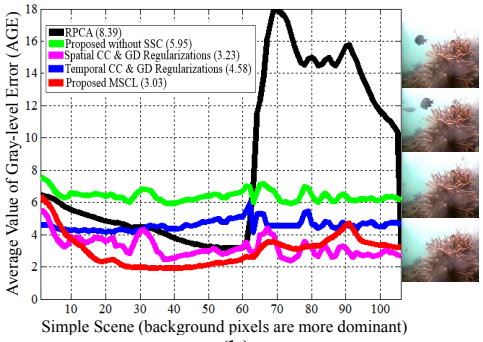

(b)

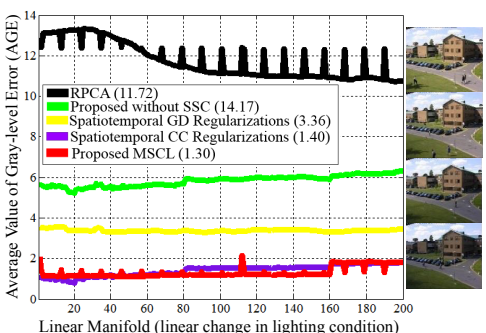

(e)

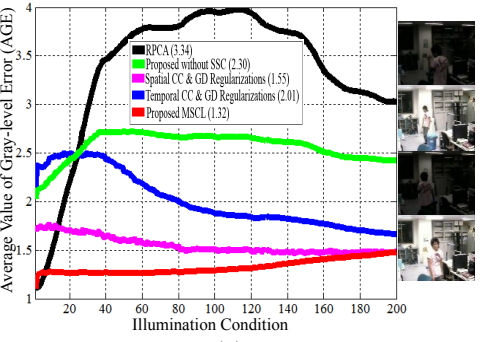

(c)

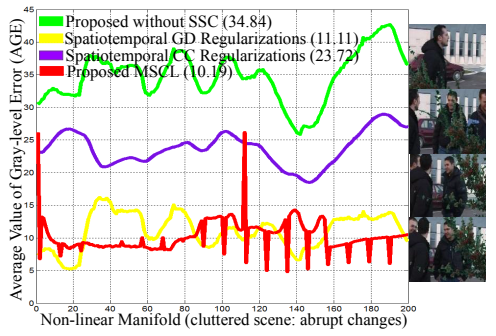

(f)

Fig. 9. Performance of estimated background model with only RPCA, spatial regularization, only temporal regularization, only correlation coefficient based spatiotemporal regularizations, only geodesic distance based spatiotemporal regularizations, and without spatiotemporal sparse subspace clustering.

TABLE I

COMPARISON OF AVERAGE F1 SCORE ON CDNET DATASET.

\begin{tabular}{|c|c|c|c|c|c|c|c|c|c|c|c|c|c|c|c|c|c|c|}
\hline Categories & DECOLOR & 3TD & DP-GMM & 2P-RPCA & LSD & SuBSENSE & PAWCS & BMTDL & TVRPCA & SRPCA & RMAMR & LR-FSO & $\overline{\mathrm{GFL}}$ & GoDec & $\overline{\text { GRASTA }}$ & GOSUS & MSCL & MSCL-FL \\
\hline Baseline & 0.92 & 0.88 & 0.92 & 0.92 & 0.92 & $0.95^{*}$ & 0.94 & 0.88 & 0.84 & 0.82 & 0.89 & 0.80 & 0.83 & 0.77 & 0.66 & 0.90 & 0.87 & 0.94 \\
\hline Dynamic Background & 0.70 & 0.75 & 0.81 & 0.78 & 0.71 & 0.81 & 0.89 & 0.75 & 0.55 & 0.84 & 0.82 & 0.74 & 0.74 & 0.58 & 0.35 & 0.79 & 0.85 & $0.90^{*}$ \\
\hline Camera Jitter & 0.77 & 0.72 & 0.74 & 0.81 & 0.78 & 0.81 & 0.81 & 0.72 & 0.63 & 0.78 & 0.75 & 0.76 & 0.78 & 0.48 & 0.43 & 0.82 & 0.83 & $0.86^{*}$ \\
\hline Shadow & 0.83 & 0.68 & 0.81 & 0.80 & 0.81 & $0.89^{*}$ & $0.89^{*}$ & 0.81 & 0.71 & 0.77 & 0.73 & 0.69 & 0.82 & 0.51 & 0.52 & 0.84 & 0.82 & 0.86 \\
\hline Thermal & 0.70 & 0.78 & 0.81 & 0.76 & 0.75 & 0.81 & 0.83 & 0.79 & 0.69 & 0.79 & 0.75 & 0.80 & 0.76 & 0.62 & 0.42 & 0.80 & 0.82 & $0.86^{*}$ \\
\hline Intermittent Object Motion & 0.59 & 0.55 & 0.54 & 0.65 & 0.67 & 0.65 & 0.77 & 0.69 & 0.57 & 0.80 & 0.66 & 0.63 & 0.59 & 0.38 & 0.35 & 0.74 & 0.80 & $0.84^{*}$ \\
\hline Bad Weather & 0.76 & 0.79 & 0.82 & 0.75 & 0.79 & 0.86 & 0.81 & 0.77 & 0.78 & 0.75 & 0.70 & 0.79 & 0.76 & 0.66 & 0.68 & 0.77 & 0.83 & $0.88^{*}$ \\
\hline Average & 0.75 & 0.73 & 0.77 & 0.78 & 0.77 & $\overline{0.82}$ & 0.84 & 0.77 & 0.68 & 0.79 & 0.75 & 0.74 & 0.75 & 0.57 & 0.48 & 0.80 & 0.83 & $\overline{0.88^{*}}$ \\
\hline
\end{tabular}

the simple case (Fig.9(b)), the spatial constraints have mostly performed better. In varying lighting conditions (Fig.9(c)), the spatial constraints performed better because they were not affected by the abrupt intensity variations. In the case of jitter (Fig.9(d)), the spatial constraints performed better because of the abrupt motion of the camera, which degraded the interframe similarity, but retained the high intra-frame similarity. In the case of linear light intensity variations (Fig.9(e)), the correlation performed better, while in the case of nonlinear variations with abrupt changes (Fig.9(f)), the geodesic distance performed better because in this case, the correlation coefficient was not able to handle the nonlinear changes. Therefore, in some cases, spatial regularization performed better, while in other cases, the performance of temporal regularization was better. However, the proposed MSCL algorithm integrated the strengths of different regularization schemes and achieved better performance compared to the individual regularization.

\section{Evaluation of MSCL-FL for Foreground Detection}

The main objective of the MSCL algorithm is to extract the background model for complex scenes. However, we have also observed improvements in the foreground detection. In this section, we compare the performance of MSCL and MSCLFL algorithms with 20 existing methods on three publicly available datasets using the $F_{1}$ score as the performance measure [17], [15], [65].

1) CDnet 2014 dataset [50] consists of eleven categories of videos and provides ground truth for each sequence. We only test seven challenging categories, namely, 'Baseline', 'Dynamic Background', 'Camera Jitter', 'Intermittent Object Motion', 'Thermal', 'Shadows', and 'Bad Weather'. These videos were captured using low-resolution IP cameras as well as thermal cameras. The spatial resolution varies from $320 \times 240$ to $720 \times 576$. The level of noise and compression artifacts also vary across videos. Table I shows the quantitative results of all the compared methods. Fig. 10 presents the visual results of the proposed MSCL-FL algorithm only.

The category Baseline contains four simple videos (Fig.10a). As shown in Table I, all of the compared methods (excluding GRASTA) produce an average $F_{1}$ score of around $90 \%$. Therefore, the Baseline category does not pose a challenge for most of the compared methods.

The Dynamic Background category contains six challenging videos (Fig.10b) depicting outdoor scenes. This is the most difficult among all categories for mounted camera object detection, which contains sequences exhibiting dynamic background motions. Most state-of-the-art methods including DECOLOR, 3TD, 2P-RPCA, LSD, and BMDTL, generate noisy foreground segments because of highly dynamic background regions. Therefore, the performance of these methods degraded compared to the proposed MSCL-FL algorithm (Table I). Given that no additional constraints are considered on the low-rank matrix; therefore, the FL-based methods, such as LR-FSO and GFL, also show a degraded performance (Table I), while very few methods, such as DP-GMM and RMAMR, attain a comparable performance of $80 \%$. The PAWCS and the proposed algorithms MSCL and MSCL-FL are the best performers for these dynamic sequences. The proposed spatiotemporal continuity in the MSCL algorithm improves the foreground detection among all the compared algorithms. However, the fusion of neighboring information 

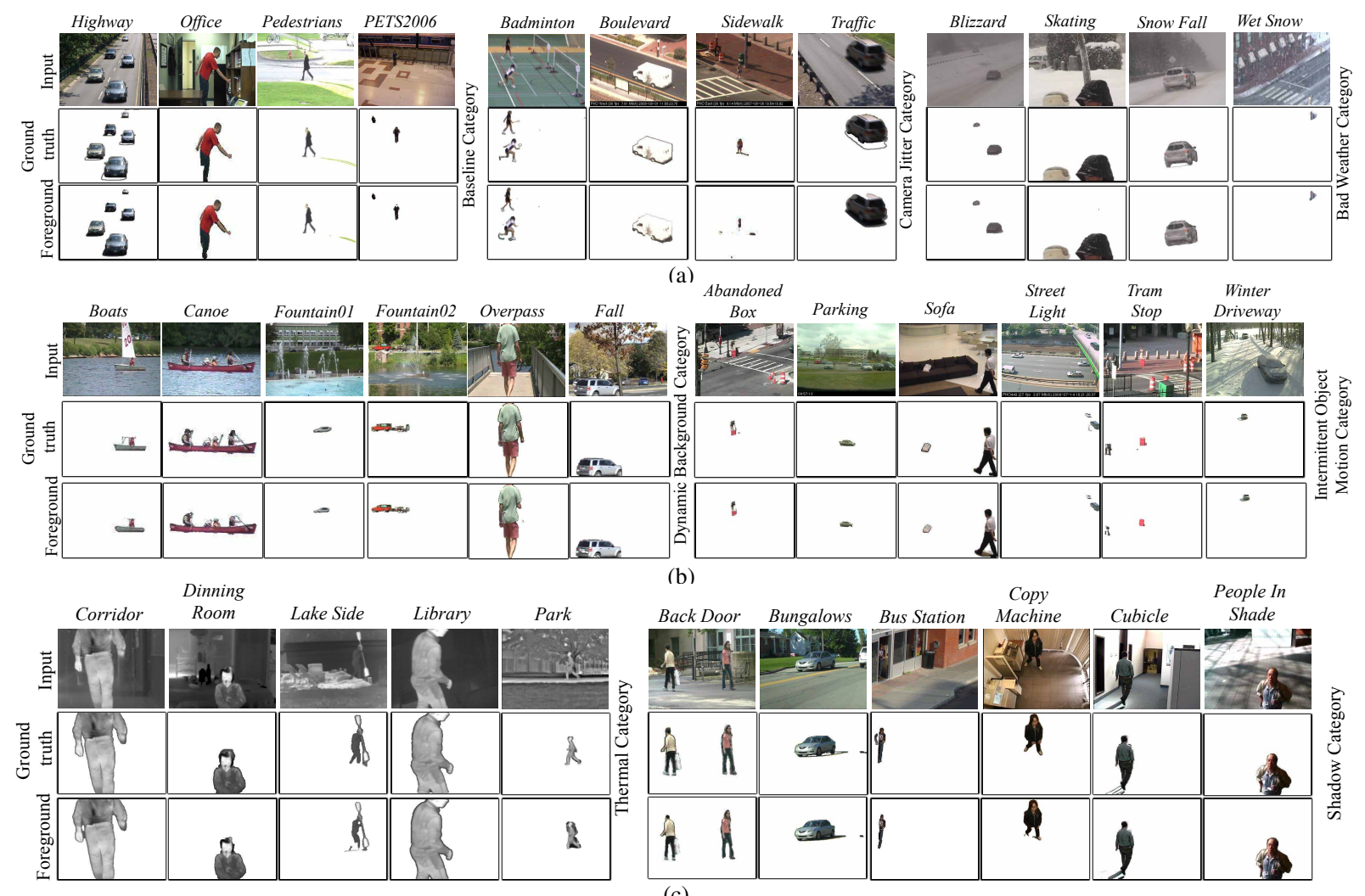

Fig. 10. Qualitative results of the proposed MSCL-FL algorithm on each category of CDnet 2014 dataset [50]. From left to right: the visual results of (a) Baseline, Camera Jitter, and Bad Weather categories, (b) Dynamic Background and Intermittent Object Motion categories, and (c) Thermal and Shadow category.

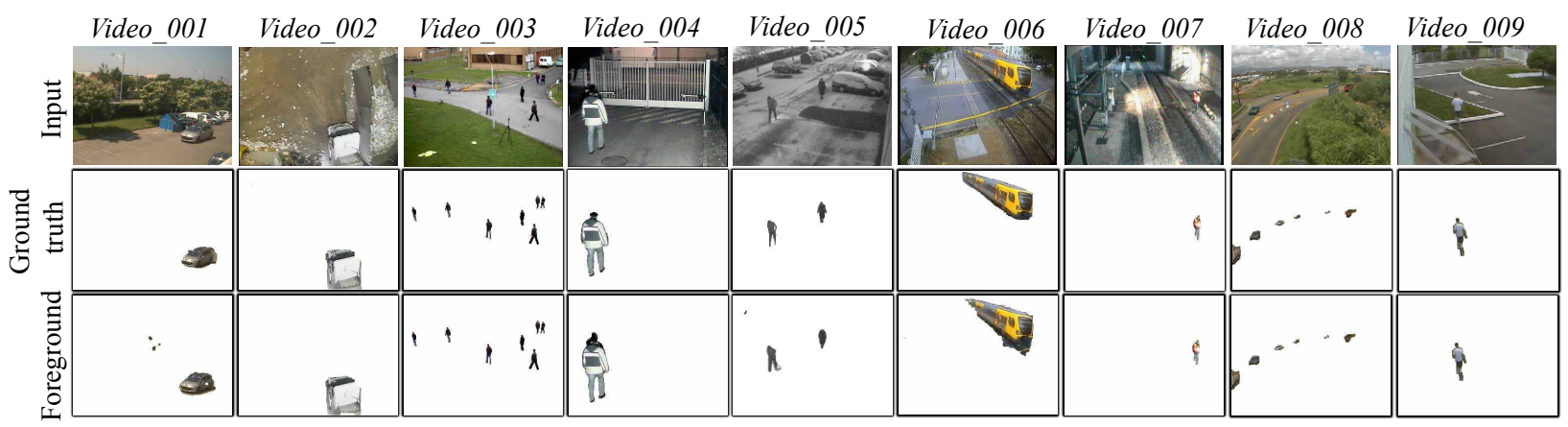

Fig. 11. Visual results of the proposed MSCL-FL algorithm over 9 sequences of BMC dataset [49]. From left to right: 9 videos are presented. From top to bottom: input frames, ground truth images, and visual results of the proposed algorithm are presented.

in the proposed MSCL-FL algorithm improves by $4 \%$ of the $F_{1}$ score. The proposed MSCL-FL algorithm achieves $90 \%$ average $F_{1}$ score, which is larger than the current state-ofthe-art methods.

The category Camera Jitter contains one indoor and three outdoor videos (Fig.10a). The compared methods generate noisy foreground mask resulting in a low $F_{1}$ score because of jitter. One of the strengths of the MSCL-FL is to handle effectively the fast motion of the background caused by camera jitter and fast moving foreground objects. The comparison between MSCL-FL and GOSUS (structured sparsity constraints with RPCA) demonstrates that the performance gained by the proposed method is not a general feature of RPCA, but it is a combined result of the spatiotemporal regularization, the optimization method, and the foreground pixel labeling process using FL. The Shadows category comprises six videos (Fig.10c) exhibiting both strong and faint shadows. For 3TD, LR-FSO, GoDec, and GRASTA methods, this category poses a big challenge (TableI). SuBSENSE, PAWCS, and MSCL-FL have achieved promising performance as compared to other methods. We observe that some hard shadows on the ground (Fig.10c, Cubicle sequence) are still a major limitation of the top performing algorithms. The Thermal category consists of five sequences captured by the far-IR camera (Fig.10c). Color saturation is the main challenge in this category, which degrades the performance of DECOLOR, 2P-RPCA, TVRPCA, RMAMR, GoDec, LSD, RMAMR, GFL, and GRASTA methods (TableI). The spatiotemporal regularization combined with the FL constraint in MSCL-FL are able to discriminate the background-foreground pixels effectively in the presence of color saturation.

The Intermittent Object Motion category includes six videos (Fig.10b), which contain ghosting artifacts in the detected motion. All compared methods except SRPCA were not able to handle this challenge and obtained a low $F_{1}$ score. The proposed MSCL-FL was able to handle the challenge of ghosting 
TABLE II

COMPARISON OF AVERAGE $\mathrm{F}_{1}$ SCORE ON EACH VIDEO OF BMC 2012 DATASET [49] (SEE FIG. 11)

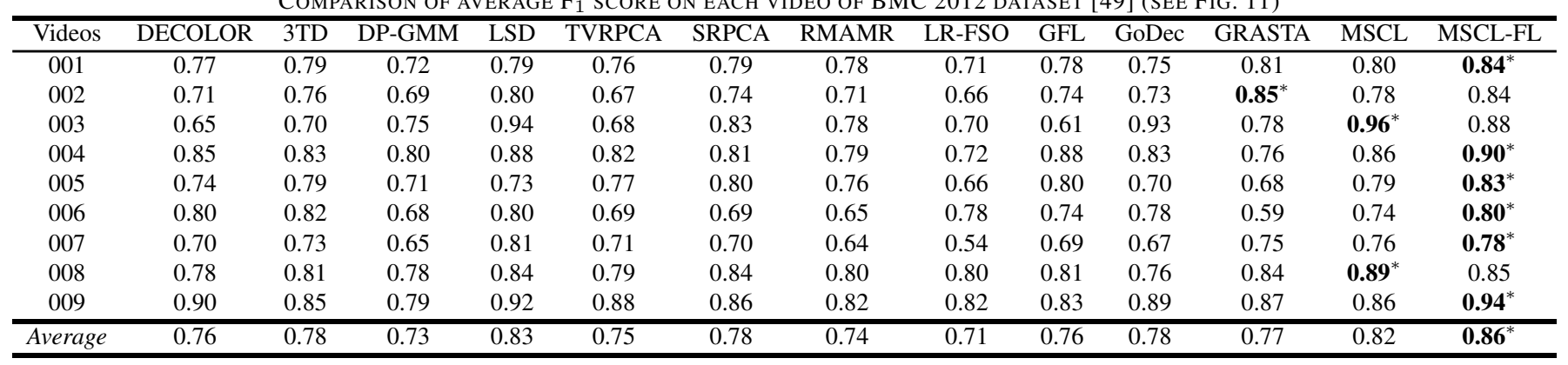

TABLE III

COMPARISON OF AVERAGE $F_{1}$ SCORE ON I2R [26] AND WALLFLOWER [48] DATASETS. BOLD FACE NUMBER REPRESENTS BEST PERFORMING METHOD.

\begin{tabular}{|c|c|c|c|c|c|c|c|c|c|c|c|c|c|c|c|c|}
\hline Datasets & $\overline{\mathrm{DECOLOR}}$ & $3 \mathrm{TD}$ & DP-GMM & LSD & TLSFSD & TVRPCA & SRPCA & RMAMR & LR-FSO & MLRSBS & MODSM & GFL & RFSA & GRASTA & MSCL & $\overline{\text { MSCL-FL }}$ \\
\hline$\overline{\mathrm{I} 2 \mathrm{R}}$ & 0.74 & $\overline{0.72}$ & 0.70 & 0.75 & 0.76 & 0.69 & 0.80 & 0.75 & 0.69 & 0.76 & 0.76 & $0.85^{*}$ & 0.71 & 0.54 & 0.82 & 0.84 \\
\hline Wallflower & 0.59 & 0.75 & 0.78 & 0.75 & N/A & 0.61 & 0.85 & 0.80 & 0.74 & N/A & 0.73 & 0.84 & 0.54 & 0.33 & 0.86 & $0.92^{*}$ \\
\hline
\end{tabular}

artifact effectively. The large margin obtained by the proposed algorithms is attributed to the removal of the motionless frames and the encoding of spatiotemporal regularization in the lowrank background model. The Bad Weather category contains six videos (Fig.10a). The moving rain streaks or snowflakes must remain part of the background model. All compared methods except SuBSENSE and PAWCS were not able to handle this challenge efficiently. In the proposed MSCL-FL algorithm, the information of neighboring pixels was useful in handling the isolated moving parts of the background, such as snowflakes and rain streaks.

On the average, on CDnet dataset, the MSCL-FL obtained $88 \% F_{1}$ score, which is significantly larger than the existing best-performing methods including GOSUS, SuBSENSE, and PAWCS. The performance of other methods has remained low (Table I).

2) Background Models Challenge (BMC) 2012 Dataset: In BMC dataset [49], we compare our results on 9 real videos including challenges, such as the presence of dynamic backgrounds, cast shadows, lighting conditions, intermittent foreground object motion, and bootstrapping. For fair comparison, the automatic evaluation tool by the original authors is used to compute the $F_{1}$ score. Fig. 11 presents the visual results of the proposed MSC-FL algorithm. Table II shows the average $F_{1}$ score of 11 compared methods. The MSCLFL algorithm has achieved the best performance over videos 001,004,005,006,007, and 009, while the MSCL algorithm obtained the best results for 003 and 008. These results are attributed to the small size of the foreground objects, which hindered FL from distinguishing these pixels efficiently. The average $F_{1}$ score obtained by the proposed MSCL-FL is $86 \%$, LSD is $83 \%$, and MSCL is $82 \%$, while all other compared methods are significantly lower.

3) I2R Dataset: We have also reported the performance of the proposed algorithms on the I2R dataset [26]. This dataset comprises 9 complex background videos including crowded foreground (Bootstrap, Shopping Mall, Escalator, and Airport Hall), dynamic background (Campus, Curtain, Fountain, and Water Surface), and drastic illumination variations (lobby). The proposed algorithms are compared with 14 state-of-theart methods in Table III. On the average, GFL obtained the best performance of 0.85 while our proposed MSCL-FL obtained 0.84 . All other compared methods exhibited degraded performance for these videos. In some cases, the MSCL performs better than MSCL-FL because of the over-smoothing induced by the FL.

4) Wallflower Dataset [48] consists of 6 challenging videos including Waving Trees, Moved Object, Light Switch, Time of Day, Camouflage, and Foreground Aperture. We compare our proposed algorithms with 12 state-of-the-art methods. On the average, the MSCL-FL outperformed the existing algorithms by a significant margin (TableIII). The GFL obtained significantly low performance of 0.84 compared to 0.92 obtained by MSCL-FL. It is because in the presence of camouflage sequence GFL failed to accurately detect moving foreground objects (Fig.3 in [54]). Also in the bootstrap sequence because of cluttered foreground objects GFL suffered from oversmoothing. The closely located distinct objects were merged into single object. While the proposed MSCL-FL algorithm was able to obtain good performance in all cases.

\section{Execution Time Comparison}

Execution times are compared on a machine with Intel core i7 processor and 8GB RAM. The computational cost of our proposed algorithm is mainly determined by the LADMAP [28]. Let $k$ denote the number of iterations and $r$ be the lowest rank for matrix $\mathbf{B}$. The construction of spatiotemporal CC-based graph Laplacians needs $O\left(p^{2} c+p c^{2}\right)$. For spatiotemporal GD-based graph Laplacians, we use Johnson's algorithm [23], which requires $O(c \log (c)+p \log (p)+(c+p) e)$ time complexity, where $e$ denotes edges. In each iteration for solving Alg. (1), SVT is applied to update the low-rank matrix B whose total complexity is $O\left(r c^{2}\right)$ when we use partial SVD. Similarly, the soft thresholding operation to update the matrix F has a complexity of $O(p c)$. Therefore, the cost of LADMAP iterations is $O\left(k r c^{2}+k p c\right)$. The dominant computational complexity is $O\left(p^{2} c\right)$ if $p>c$ and $O\left(p c^{2}\right)$ if $c>p$. We have also compared the computational time of the proposed MSCL algorithm. For this purpose, we selected a highway sequence with 50 frames of size $320 \times 240$. The overall time taken by MSCL is $39.8 \mathrm{sec}$, which includes $20 \mathrm{sec}$ taken by the optical flow computation and $19.8 \mathrm{sec}$ by the rest of the algorithm. On 
the same sequence, GRASTA, DECOLOR, RFSA, RMAMR, 3TD, BSLSD, and GFL took 86, 291, 72, 30, 106, 71, and 66 $\mathrm{sec}$, respectively. The execution time of the proposed algorithm can be further enhanced by using GPU or CPU based parallel implementation. Moreover, a faster optical flow computation method will also make the overall process faster.

\section{CONCLUSion}

In this study, an algorithm based on RPCA with spatiotemporal sparse spectral clustering based regularization is presented for efficient background modeling. To reduce outliers, motionless frames are removed. The regularization enforced the background model to be continuous on the low dimensional multiple manifolds both in the spatial and temporal dimensions, which is achieved by constructing two graphs to encode the temporal and spatial similarities. The objective function is efficiently solved using LADMAP. The major advantage of the proposed algorithm is its capacity to generate an accurate background model even in the presence of occlusions, clutter, jitter, and abrupt intensity variations. Large scale experimental evaluations on five datasets demonstrated that the proposed algorithm achieved the best performance compared to existing methods. However, videos captured from moving and PTZ cameras and online processing remain open challenges. We plan to investigate the possibility of extending the proposed algorithm to scenes that are more crowded and to those recorded using a moving camera by further extending the notion of data similarity using coarse-to-fine strategy.

\section{ACKNOWLEDGMENTS}

This work was supported by the National Research Foundation of Korea funded by the Korean Government (NRF20170915).

\section{REFERENCES}

[1] A. Beck and M. Teboulle, "A fast iterative shrinkage-thresholding algorithm for linear inverse problems," SJIS, vol. 2, no. 1, pp. 183-202, 2009.

[2] T. Bouwmans, L. Maddalena, and A. Petrosino, "Scene background initialization: A taxonomy," PRL, 2017.

[3] T. Bouwmans, A. Sobral, S. Javed, S. K. Jung, and E.-H. Zahzah, "Decomposition into Low-rank plus additive matrices for background/foreground separation: A review for a comparative evaluation with a large-scale dataset," CSR, 2016.

[4] T. Bouwmans and E. H. Zahzah, "Robust PCA via principal component pursuit: a review for a comparative evaluation in video surveillance," CVIU, vol. 122, pp. 22-34, 2014

[5] M. Braham and M. Van Droogenbroeck, "Deep background subtraction with scene-specific convolutional neural networks," in IEEE Sys., Sig. and IP. IEEE, 2016, pp. 1-4.

[6] J.-F. Cai, E. J. Candès, and Z. Shen, "A singular value thresholding algorithm for matrix completion," S. J. on Opt., vol. 20, no. 4, pp. 1956-1982, 2010.

[7] E. J. Candès, X. Li, Y. Ma, and J. Wright, "Robust principal component analysis?" JACM, vol. 58, no. 3, p. 11, 2011.

[8] X. Cao, L. Yang, and X. Guo, "Total Variation Regularized RPCA for Irregularly Moving Object Detection Under Dynamic Background," IEEE T-C, vol. 46, no. 4, pp. 1014-1027, April 2016.

[9] M. Chen, X. Wei, Q. Yang, Q. Li, G. Wang, and M. H. Yang, "Spatiotemporal gmm for background subtraction with superpixel hierarchy," IEEE T-PAMI, vol. PP, no. 99, pp. 1-1, 2017.

[10] A. da Miranda Neto, "Pearson's Correlation Coefficient: A More Realistic Threshold for Applications on Autonomous Robotics," CTA, vol. 5, no. 2,2014
[11] O. Diego, C. S. M. Juan, and M. M. Jose, "Rejection based multipath reconstruction for background estimation in video sequences with stationary objects," CVIU, vol. 147, pp. 23-37, 2016.

[12] E. Elhamifar and R. Vidal, "Sparse subspace clustering: Algorithm, theory, and applications," IEEE T-PAMI, vol. 35, no. 11, pp. 2765-2781, 2013.

[13] N. B. Erichson and C. Donovan, "Randomized low-rank Dynamic Mode Decomposition for motion detection," CVIU, vol. 146, pp. 40-50, 2016.

[14] G. Gallo, M. D. Grigoriadis, and R. E. Tarjan, "A fast parametric maximum flow algorithm and applications," SIAM J. on Comp., vol. 18, no. 1, pp. 30-55, 1989.

[15] Z. Gao, L.-F. Cheong, and Y.-X. Wang, "Block-sparse RPCA for salient motion detection," IEEE T-PAMI, vol. 36, no. 10, pp. 1975-1987, 2014.

[16] X. Guo, X. Wang, L. Yang, X. Cao, and Y. Ma, "Robust foreground detection using smoothness and arbitrariness constraints," in Eccv. Springer, 2014

[17] T. S. Haines and T. Xiang, "Background subtraction with DirichletProcess mixture models," IEEE T-PAMI, vol. 36, no. 4, pp. 670-683, 2014.

[18] J. He, L. Balzano, and A. Szlam, "Incremental gradient on the grassmannian for online foreground and background separation in subsampled video," in CVPR. IEEE, 2012.

[19] W. Hu, Y. Yang, W. Zhang, and Y. Xie, "Moving object detection using tensor-based low-rank and saliently fused-sparse decomposition," IEEE T-IP, vol. 26, no. 2, pp. 724-737, Feb 2017.

[20] S. Javed, A. Mahmood, T. Bouwmans, and S. K. Jung, "MotionAware Graph Regularized RPCA for Background Modeling of Complex Scenes," in ICPR-CP, 2016.

[21] S. Javed, S. H. Oh, T. Bouwmans, and S. K. Jung, "Robust background subtraction to global illumination changes via multiple features-based online robust principal components analysis with markov random field," $J E I$, vol. 24, no. 4, pp. 043011-043011, 2015.

[22] X. Jiang, H. Wang, S. Tang, L. Ma, Z. Zhang, and Z. Zheng, "A new approach to shortest paths on networks based on the quantum bosonic mechanism," N. JP, vol. 13, no. 1, p. 013022, 2011.

[23] D. B. Johnson, "Efficient algorithms for shortest paths in sparse networks," JACM, vol. 24, no. 1, pp. 1-13, 1977.

[24] R. M. Larsen, "Lanczos bidiagonalization with partial reorthogonalization," D. R. S., vol. 27, no. 537, 1998.

[25] H. Lee, H. Kim, and J. I. Kim, "Background Subtraction Using Background Sets with Image- and Color-Space Reduction," IEEE T-M, vol. 18, no. 10, pp. 2093-2103, Oct 2016.

[26] L. Li, W. Huang, I. Y.-H. Gu, and Q. Tian, "Statistical modeling of complex backgrounds for foreground object detection," IEEE T-IP, vol. 13, no. 11, pp. 1459-1472, 2004.

[27] Z. Lin, M. Chen, and Y. Ma, "The augmented Lagrange multiplier method for exact recovery of corrupted low-rank matrices," arXiv preprint arXiv:1009.5055, 2010.

[28] Z. Lin, R. Liu, and Z. Su, "Linearized alternating direction method with adaptive penalty for low-rank representation," in NIPS, 2011.

[29] C. Liu, "Beyond pixels: exploring new representations and applications for motion analysis," Ph.D. dissertation, Citeseer, 2009.

[30] R. Liu, Z. Lin, and Z. Su, "Linearized alternating direction method with parallel splitting and adaptive penalty for separable convex programs in machine learning." in ACML, 2013.

[31] X. Liu, G. Zhao, J. Yao, and C. Qi, "Background subtraction based on low-rank and structured sparse decomposition," IEEE T-IP, vol. 24, no. 8, pp. 2502-2514, 2015.

[32] L. Maddalena and A. Petrosino, "Towards benchmarking scene background initialization," in ICIAP. Springer, 2015.

[33] A. Mahmood and S. Khan, "Exploiting transitivity of correlation for fast template matching," IEEE T-IP, vol. 19, no. 8, pp. 2190-2200, 2010.

[34] A. Mahmood, A. Mian, and R. Owens, "Semi-supervised spectral clustering for image set classification," in cvpr, 2014.

[35] O. Oreifej, X. Li, and M. Shah, "Simultaneous video stabilization and moving object detection in turbulence," IEEE T-PAMI, vol. 35, no. 2, pp. 450-462, 2013.

[36] Y. Pang, L. Ye, X. Li, and J. Pan, "Incremental learning with saliency map for moving object detection," IEEE T-CSVT, vol. PP, no. 99, pp. $1-1,2016$.

[37] J. Pierre-Marc, M. Lucia, and P. Alfredo, "Scene Background Modeling.net," in $I C P R, 2016$.

[38] M. Qin, Y. Lu, H. Di, and W. Huang, "A background basis selectionbased foreground detection method," IEEE T-M, vol. 18, no. 7, pp. 12831296, July 2016.

[39] J. Sajid, M. Arif, B. Thierry, and J. S. Ki, "Spatiotemporal low-rank modeling for complex scene background initialization," IEEE T-CSVT, 2016. 
[40] N. Shahid, V. Kalofolias, X. Bresson, M. Bronstein, and P. Vandergheynst, "Robust principal component analysis on graphs," in ICCV, 2015.

[41] A. Shimada, H. Nagahara, and R.-i. Taniguchi, "Background modeling based on bidirectional analysis," in IEEE CVPR, 2013, pp. 1979-1986.

[42] P. L. St-Charles, G. A. Bilodeau, and R. Bergevin, "Universal Background Subtraction Using Word Consensus Models," IEEE T-IP, vol. 25, no. 10 , pp. 4768-4781, Oct 2016.

[43] P.-L. St-Charles, G.-A. Bilodeau, and R. Bergevin, "Subsense: A universal change detection method with local adaptive sensitivity," IEEE T-IP, vol. 24, no. 1, pp. 359-373, 2015.

[44] A. Staglianò, N. Noceti, A. Verri, and F. Odone, "Online space-variant background modeling with sparse coding," IEEE T-IP, vol. 24 , no. 8 , pp. 2415-2428, 2015.

[45] C. Stauffer and W. E. L. Grimson, "Adaptive background mixture models for real-time tracking," in IEEE CVPR, vol. 2, 1999, pp. 246-252.

[46] R. Tibshirani, "Regression shrinkage and selection via the lasso," $J$. $R$. S. S. S. B (M.), pp. 267-288, 1996.

[47] R. Tibshirani, M. Saunders, S. Rosset, J. Zhu, and K. Knight, "Sparsity and smoothness via the fused lasso," J. of R. S. S.: S. B (S. M.), vol. 67, no. 1, pp. 91-108, 2005.

[48] K. Toyama, J. Krumm, B. Brumitt, and B. Meyers, "Wallflower: Principles and practice of background maintenance," in ICCV, vol. 1. IEEE, 1999.

[49] A. Vacavant, T. Chateau, A. Wilhelm, and L. Lequièvre, "A benchmark dataset for outdoor foreground/background extraction," in ACCV. Springer, 2012.

[50] Y. Wang, P.-M. Jodoin, F. Porikli, J. Konrad, Y. Benezeth, and P. Ishwar, "Cdnet 2014: an expanded change detection benchmark dataset," in IEEE CVPRW, 2014.

[51] Y. Wang, Z. Luo, and P.-M. Jodoin, "Interactive deep learning method for segmenting moving objects," PRL, 2016.

[52] Z. Wen, D. Goldfarb, and W. Yin, "Alternating direction augmented Lagrangian methods for semidefinite programming," $M P C$, vol. 2, no. 3-4, pp. 203-230, 2010.

[53] J. Wright, A. Ganesh, S. Rao, Y. Peng, and Y. Ma, "Robust principal component analysis: Exact recovery of corrupted low-rank matrices via convex optimization," in NIPS, 2009, pp. 2080-2088.

[54] B. Xin, Y. Tian, Y. Wang, and W. Gao, "Background subtraction via generalized fused lasso foreground modeling," in CVPR, 2015.

[55] J. Xu, V. Ithapu, L. Mukherjee, J. Rehg, and V. Singh, "Gosus: Grassmannian online subspace updates with structured-sparsity," in ICCV, 2013.

[56] G. Xue, L. Song, and J. Sun, "Foreground estimation based on linear regression model with fused sparsity on outliers," IEEE T-CSVT, vol. 23, no. 8, pp. 1346-1357, 2013.

[57] X. Ye, J. Yang, X. Sun, K. Li, C. Hou, and Y. Wang, "ForegroundBackground Separation From Video Clips via Motion-Assisted Matrix Restoration," IEEE T-CSVT, vol. 25, no. 11, pp. 1721-1734, Nov 2015.

[58] M. Yin, J. Gao, Z. Lin, Q. Shi, and Y. Guo, "Dual graph regularized latent low-rank representation for subspace clustering," IEEE T-IP, vol. 24, no. 12, pp. 4918-4933, 2015.

[59] T. Zhang, S. Liu, N. Ahuja, M.-H. Yang, and B. Ghanem, "Robust visual tracking via consistent low-rank sparse learning," IJCV, vol. 111, no. 2, pp. 171-190, 2015.

[60] T. Zhang, S. Liu, C. Xu, and H. Lu, "Mining semantic context information for intelligent video surveillance of traffic scenes," IEEE T-II, vol. 9, no. 1, pp. 149-160, 2013.

[61] Y. Zhang, X. Li, Z. Zhang, F. Wu, and L. Zhao, "Deep learning driven blockwise moving object detection with binary scene modeling," Neurocomputing, vol. 168, pp. 454-463, 2015.

[62] Z. Zhang and K. Zhao, "Low-rank matrix approximation with manifold regularization," IEEE T-PAMI, vol. 35, no. 7, pp. 1717-1729, 2013.

[63] Q. Zhao, G. Zhou, L. Zhang, A. Cichocki, and S.-I. Amari, "Bayesian Robust Tensor Factorization for Incomplete Multiway Data," IEEE TNNLS, vol. PP, no. 99, pp. 1-1, 2016.

[64] T. Zhou and D. Tao, "Godec: Randomized low-rank and sparse matrix decomposition in noisy case," in ICML. Omnipress, 2011.

[65] X. Zhou, C. Yang, and W. Yu, "Moving object detection by detecting contiguous outliers in the low-rank representation," IEEE T-PAMI, vol. 35, no. 3, pp. 597-610, 2013.

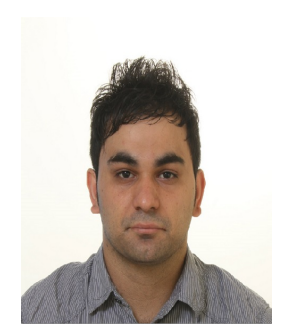

Sajid Javed received the B.Sc. (Hons.) degree in computer science from University of Hertfordshire, U.K, in 2010. He joined the virtual reality laboratory in Kyungpook National University, Republic of Korea, in 2012, from where he completed the integrated Masters and Ph.D. degree in computer science in 2017. He is now a Post-doctoral fellow in University of Warwick, U.K, where he is pursuing his future research on medical image analysis under the supervision of Prof. Nasir Rajpoot. He has an active collaboration with the MIA Laboratory, France. His research interests include RPCA, matrix completion, dictionary learning, deep learning, background modeling, foreground detection, background subtraction, and subspace clustering.

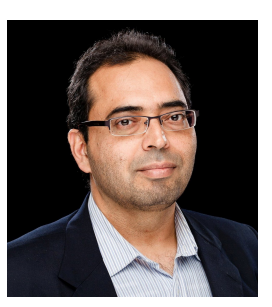

Arif Mahmood received his Masters and the Ph.D. degrees in Computer Science from Lahore University of Management Sciences, Lahore, Pakistan in 2003 and 2011 respectively. Currently he is Postdoc researcher in the Department of Computer Science and Engineering, Qatar University, Doha. His current research directions are action detection and person segmentation in crowded environments, and background-foreground modeling in complex scenes. Before this he worked as Research Assistant Professor with the School of Mathematics and Statistics, the University of Western Australia. He worked on characterizing structure of complex networks using sparse subspace clustering. Before that he was Research Assistant Professor with the School of Computer Science and Software Engineering, UWA and worked on hyper-spectral object recognition and action recognition using depth images. His major research interests are in Computer Vision and Pattern Recognition. More specifically he has performed research in data clustering, classification, action and object recognition.

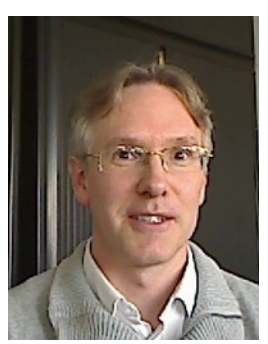

Thierry Bouwmans is an associate professor at the University of La Rochelle, France. His research interests include detection of moving objects. $\mathrm{He}$ is the creator and administrator of the background subtraction web site. He served as the lead guest editor in two editorial works: (1) Special issue in MVA on background modeling for foreground detection in real world dynamic scenes, (2) Handbook on Background Modeling and Foreground Detection in CRC Press.

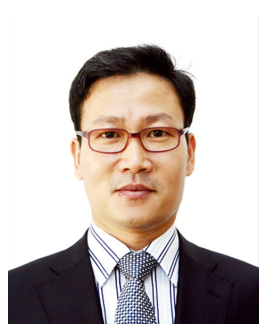

Soon Ki Jung is a professor in the School of Computer Science and Engineering at Kyungpook National University, Republic of Korea. He received his $\mathrm{MS}$ and $\mathrm{PhD}$ degrees in computer science from Korea Advanced Institute of Science and Technology (KAIST), Korea, in 1992 and 1997, respectively. He has been a visiting professor at University of Southern California, USA, in 2009. He has been an active executive board member of Human Computer Interaction, Computer Graphics, and Multimedia societies in Korea. Since 2007, he has also served as executive board member of IDIS Inc. His research areas include a broad range of computer vision, computer graphics, and virtual reality topics. 